\title{
Epiphytic diatoms (Diatomeae) from Piraquara II urban reservoir, Paraná state
}

\author{
Raquel Cristina Marra ${ }^{1}$, Priscila Izabel Tremarin', Vanessa Majewski Algarte ${ }^{I}$ \& Thelma Veiga Ludwig ${ }^{\text {” }}$ \\ ${ }^{1}$ Universidade Federal do Paraná, Curitiba, PR, Brazil. \\ * Corresponding author: veigaufpr@gmail.com
}

MARRA, R.C., TREMARIN, P.I., ALGARTE, V.M., LUDWIG, T.V. Epiphytic diatoms (Diatomeae) from Piraquara II urban reservoir, Paraná state. Biota Neotropica. 16(4): e20160200. http://dx.doi.org/10.1590/1676-0611-BN-2016-0200

\begin{abstract}
We conducted a taxonomical study of epiphytic diatoms on the macrophytes Polygonum hydropiperoides, Ludwigia peruviana and Alternanthera philoxeroides collected in the mesotrophic reservoir Piraquara II flooded in 2009, located in the state of Paraná. A total of 135 infrageneric taxa were identified, among them five at generic level and other five are first records to the state. We provided illustration, valve metrics, meristics limits and taxonomic reference for each taxon. Also, life forms and species frequency are given. The most frequent diatoms totalized $15.3 \%$ of total identified taxa and sporadic species represented 54.7\%. Achnanthidium minutissimum (Kützing) Czarnecki and Brachysira neoexilis Lange-Bertalot occurred in more than $90 \%$ of analyzed samples. Among the very frequent diatoms we found other species included in Achnantidium, Fragilaria and Eunotia. The solitary Discotella stelligera (Cleve \& Grunow) Houk \& Klee and the short chain Aulacoseira tenella (Nygaard) Simonsen are free living species that entangle among diatoms from the biofilm.
\end{abstract}

Keywords: Bacillariophyta, freshwater, macrophytes, mesotrophic, periphyton, taxonomy.

\section{Diatomáceas epifíticas (Diatomeae) no reservatório urbano Piraquara II, estado do Paraná}

Resumo: Realizamos um estudo taxonômico das diatomáceas epifíticas nas macrófitas: Polygonum hydropiperoides, Ludwigia peruviana e Alternanthera philoxeroides, coletadas no reservatório Piraquara II, uma represa urbana mesotrófica inundada em 2009, localizado no estado do Paraná. Um total de 135 táxons infragenéricos foi determinado, entre os quais cinco foram citações pioneiras para o Estado. Ilustrações, limites métricos, merísticos e referências taxonômicas para cada táxon foram providenciadas. Também, dados sobre formas de vida e frequência das espécies foram adicionados. As diatomáceas mais frequentes totalizaram 15,3\% dos táxons determinados e as espécies esporádicas representaram 54\%. Achnanthidium minutissimum (Kützing) Czarnecki and Brachysira neoexilis Lange-Bertalot ocorreram em mais de 90\% das amostras analisadas. Dentre as diatomáceas muito frequentes encontram-se outras espécies de Achnanthidium, Fragilaria e Eunotia. Discostella stelligera (Cleve \& Grunow) Houk \& Klee, uma diatomácea solitária, e Aulacoseira tenella (Nygaard) Simonsen, com cadeias curtas, são espécies livres que se emaranham entre as diatomáceas do biofilme.

Palavras-chave: Bacillariophyta, água doce, macrófitas, perifíton, taxonomia.

\section{Introduction}

Stems of emergent macrophytes are suitable colonizable surfaces to epiphyton communities and are particularly able to transfer a small amount of nutrients to their epiphytes (Cattaneo \& Kalff, 1979). As periphytic diatoms are sensitive to eutrophication, it is important to record the species that occured at present for comparision to the future assemblies, helping to understand the relationship between the species and the trophic level of aquatic system. Informations about biodiversity represent a useful tool for ecological and applied studies, but an accurate taxonomy is fundamental. Identification to species level is time consuming and sometimes difficult, but useful and necessary to future ecological studies (Kociolek 2005) in this urban reservoir.

Cetto et al. (2004), Silva et al. (2010), Bertolli et al. (2010) and Faria et al. (2010) provided recent diatom inventories from eutrophic and mesotrophic urbans reservoirs: Iraí, Passaúna and Itaqui. Also,
Faria et al. (2013) selected tolerant diatom species from Itaqui reservoir. There are no related studies in the recently flooded Piraquara II urban reservoir. Bittencourt \& Gobbi (2006) evaluated phosphorous total maximum daily load in the drainage area of Piraquara II reservoir before flooding. The study demonstrated the reservoir present high potencial to eutrophication due to the intense agricultural use of soil and shallow water. The urban reservoirs have been suffering severe anthropogenic nutrients inputs and consequently eutrophication is accelerated (Calisto et al., 2014) and periphyton may respond by changes in abundance and taxonomic composition (Stoermer \& Smol 2004).

We conducted a taxonomical study of epiphytic diatoms in Piraquara II reservoir. For each taxon, we provided illustration, valve metrics and meristics limits, occurence, and literature to species taxonomic determination. The taxa first registered to state of Paraná were also described and commented. 


\section{Materials and Methods}

Piraquara II reservoir $\left(25^{\circ} 30^{\prime} \mathrm{S}\right.$ and $\left.49^{\circ} 00^{\prime} \mathrm{W}\right)$ is located in a preservation area and was built in 2008 by the dam of Piraquara river, inserted in the Iguaçu watershed, Paraná state, south Brazil (Figure 1). This shallow reservoir with 75 days of water retention time is used for public supply (depth $3.7 \mathrm{~m}$, area $5.64 \mathrm{~km}^{2}$, drainage area $58 \mathrm{~km}^{2}$ ), and is mesotrophic most part of the year (Table 1, SANEPAR, 2013, unpublished data) with Trophic State Index (TSI) around 54 from 55 TSI (SANEPAR, 2010-2014, unpublished data). The main economic activities in the vicinity are the livestock and corn culture (Bittencourt \& Gobbi 2006).

Epiphytic diatoms were sampled in June and November 2013 from stems of the emergent macrophytes Polygnum hydropiperoides Michaux (Polygonaceae), Ludwigia peruviana (L.) H. Hara (Onagraceae) and the amphibious Alternanthera philoxeroides (Mart.) Griseb. (Amaranthaceae). The macrophytes were collected according to the local availability in six sampling sites (1-6) along the reservoir (Table 2, Figure 1), three stems $(a, b, c)$ from each core were collected. Diatoms were scrapped off the substrates surfaces with an aluminium spatule, and the samples were cleaned with $\mathrm{KMnO}_{4}$ and $\mathrm{HCl}$ (Simonsen 1974 modified by Moreira-Filho \& Valente-Moreira 1981). Light microcopy-slides were mounted with Naphrax ${ }^{\circledR}$ and analyzed under Olympus BX40 microscope. Illustrations were captured by DP71 digital equipment. Cleaned samples were prepared and analised at JEOL JSM 6360LV scanning electron microscope (Eletronic Microscopy Center - Federal University of Paraná). Valve terminology followed Barber \& Haworth (1981) and Round et al. (1990). Frequencies of occurrence were stablished based on Mateucci \& Colma (1982): species as high frequent $(\mathrm{F} \geq 70 \%)$, frequent $(40 \% \leq \mathrm{F}<70 \%)$, low frequent $(10 \% \leq \mathrm{F}<40 \%)$ and sporadic $(\mathrm{F}<10 \%)$.

The examined slides and samples were housed at the herbarium of the Universidade Federal do Paraná (UPCB) (Table 2). First recorded taxa to the state of Paraná and those identified to the generic level were described and commented. Diatoms were identified to the lowest taxonomical level according to current taxonomic literature. The references used to identify each taxon even as metrics and meristics limits are listed on Table 3.
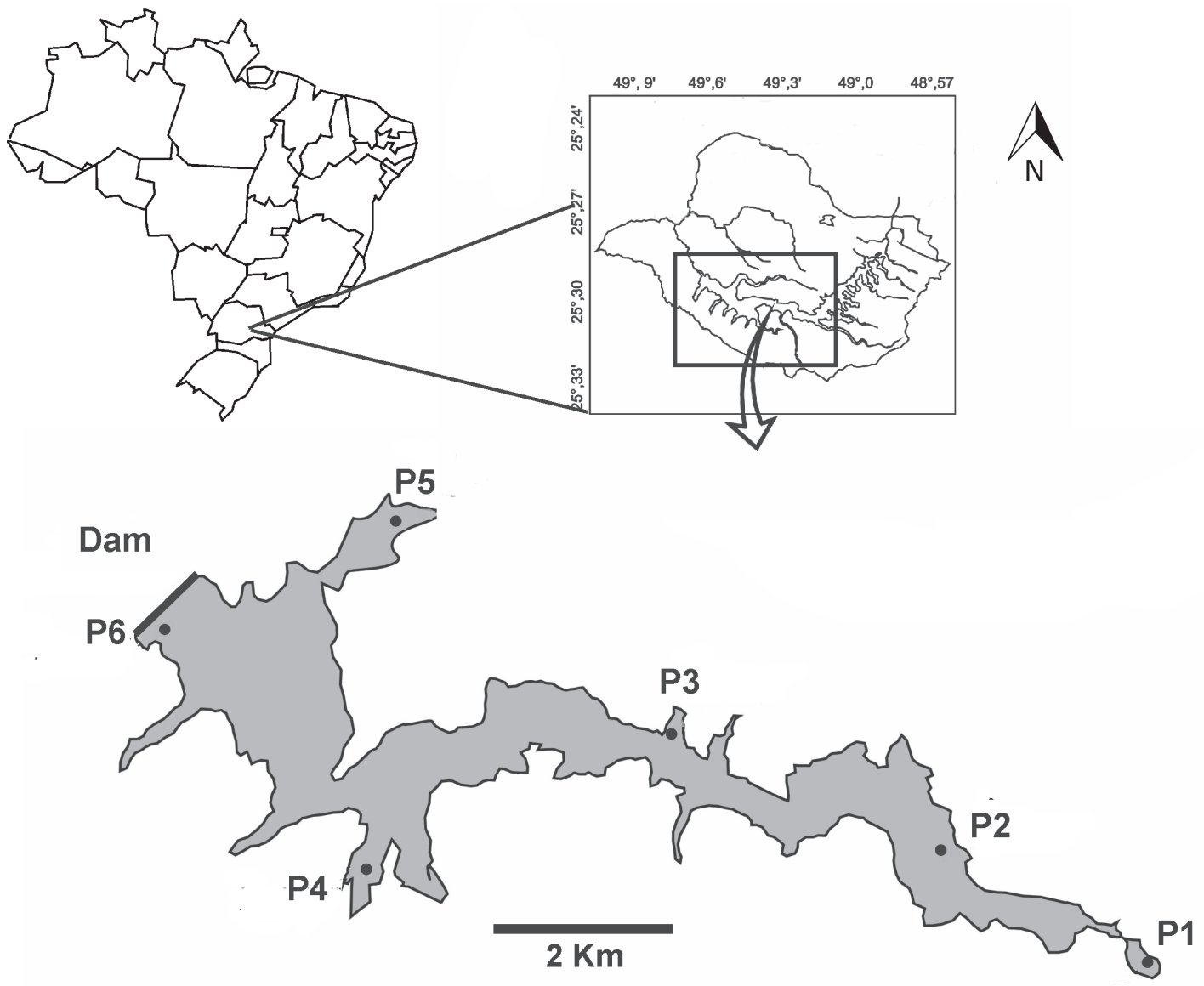

Figure 1. Localization of sampling sites in Piraquara II reservoir. Piraquara municipality, Paraná state, Brazil (modified from Google Earth 2013)

Table 1. Average set of abiotic data in the months of sampling epiphyton

\begin{tabular}{cccccccccc}
\hline Date & DO (mg/L) & Secchi $(\mathbf{m})$ & $\mathbf{p H}$ & COD $(\mathbf{m g} / \mathbf{L})$ & TN (mg/L) & TP(mg/L) & TDS (mg/L) & $\begin{array}{c}\text { Turbidity } \\
\text { (NTU) }\end{array}$ & IQA \\
\hline Jun/2013 & 6.7 & 2.7 & 6.8 & 12.8 & 1.4 & 0.025 & 46.0 & 3.0 & 84.5 \\
SD & \pm 0.3 & \pm 1.3 & \pm 0.3 & \pm 8.1 & \pm 0.3 & \pm 0 & \pm 7.0 & \pm 0.3 \\
Nov/2013 & 7.24 & 1.4 & 6.95 & 12.66 & 0.5 & 0.025 & 35.0 & 3.55 & 84,33 \\
SD & \pm 0.07 & \pm 0 & \pm 0.25 & \pm 5.43 & \pm 0 & \pm 0 & \pm 0 & \pm 0.75 & \pm 5.31 \\
\hline
\end{tabular}

*DO: dissolved oxygen; COD: Chemical oxygen demand; TN: Total Nitrogen dissolved; TP: Total phosphorus dissolved; TDS: Total dissolved solids; IQA: Index Water Quality; SD: standard deviation.

SANEPAR (2013), unpublished data 
Epiphytic diatoms from Piraquara II reservoir

Table 2. Piraquara II reservoir informations. Collected species of macrophytes, sampling sites and sample register number at Universidade Federal do Paraná Herbarium (UPCB).

\begin{tabular}{|c|c|c|c|c|}
\hline Sampling sites & Coordinates & Sampled macrophytes & \multicolumn{2}{|c|}{ UPCB } \\
\hline 1 & $\begin{array}{l}\text { S } 25^{\circ} 30^{\prime} 38.2^{\prime \prime} \\
\text { W 491'38." }\end{array}$ & Polygnum hydropiperoides & 78032 & 78044 \\
\hline 2 & $\begin{array}{l}\text { S } 25^{\circ} 30^{\prime} 23.2^{\prime \prime} \\
\text { W } 49^{\circ} 22^{\prime} 12.6^{\prime \prime}\end{array}$ & Ludwigia peruviana & 78033 & 78045 \\
\hline 3 & $\begin{array}{l}\text { S } 25^{\circ} 29^{\prime} 42.9^{\prime \prime} \\
\text { W } 49^{\circ} 3^{\prime} 28.4^{\prime \prime}\end{array}$ & $\begin{array}{l}\text { Althernanthera philoxeroides } \\
\text { Polygnum hydropiperoides }\end{array}$ & 78034 & 78046 \\
\hline 4 & $\begin{array}{l}\text { S } 25^{\circ} 30^{\prime} 18.4^{\prime \prime} \\
\text { W } 49^{\circ} 2^{\prime} 44.1^{\prime \prime}\end{array}$ & $\begin{array}{l}\text { Althernanthera philoxeroides } \\
\text { Polygnum hydropiperoides }\end{array}$ & 78035 & 78047 \\
\hline 5 & $\begin{array}{l}\text { S } 25^{\circ} 28^{\prime} 47.3^{\prime \prime} \\
\text { W } 49^{\circ} 4^{\prime} 42.1^{\prime \prime}\end{array}$ & Polygnum hydropiperoides & 78036 & 78048 \\
\hline 6 & $\begin{array}{l}\text { S } 25^{\circ} 29^{\prime} 30.1^{\prime \prime} \\
\text { W } 49^{\circ} 5^{\prime} 38.9^{\prime \prime}\end{array}$ & Polygnum hydropiperoides & 78037 & 78049 \\
\hline
\end{tabular}

Table 3. List of diatom taxa identified from Piraquara II reservoir. Morphometric \& meristics limits, occurrence in samples \& consulted literature. D: diameter ( $\mu \mathrm{m}$ ); L: length $(\mu \mathrm{m})$; W: width $(\mu \mathrm{m})$; SH: semi cell height $(\mu \mathrm{m})$; S: striae in $10 \mu \mathrm{m}$; A: areolae in $10 \mu \mathrm{m}$; F: fibulae in $10 \mu \mathrm{m}$; AC: aliform chanells in $10 \mu \mathrm{m}$.

\begin{tabular}{|c|c|c|c|c|}
\hline \multirow{2}{*}{ Species Name (Figure) } & \multirow{2}{*}{$\begin{array}{l}\text { Metric }(\mu \mathrm{m}) \& \text { meristic } \\
\quad \text { (in } 10 \mu \mathrm{m}) \text { limits }\end{array}$} & \multicolumn{2}{|c|}{ Month of occurrence at sampling sites } & \multirow{2}{*}{ Consulted literature } \\
\hline & & June & November & \\
\hline \multicolumn{5}{|l|}{ Stephanodiscaceae } \\
\hline Cyclotella meneghiniana Kützing (Figure 2) & D:9.9-15; S:10-12 & $4 \mathrm{~b}, 6 \mathrm{~b}$ & & Krammer \& Lange-Bertalot (1991a) \\
\hline $\begin{array}{l}\text { Discostella stelligera (Cleve \& Grunow) Houk } \\
\& \text { Klee (Figures 3-4) }\end{array}$ & D:7.4-13.9; S:8-13 & $\begin{array}{c}\text { 1a, } 2 \mathrm{abc}, 3 \mathrm{abc} 4 \mathrm{abc} \\
5 \mathrm{abc}, 6 \mathrm{abc}\end{array}$ & $2 \mathrm{ab}, 3 \mathrm{ab}, 4 \mathrm{ac}, 5 \mathrm{ab}, 6 \mathrm{ab}$ & Houk et al. (2010) \\
\hline \multicolumn{5}{|l|}{ Aulacoseiraceae } \\
\hline $\begin{array}{l}\text { Aulacoseira ambigua (Grunow) Simonsen var. } \\
\text { ambigua f. ambigua (Figures 15-16) }\end{array}$ & SH:7.1-12.2; D:6-7.5; S:8 & $\begin{array}{l}\text { 1ab, 2abc, 3abc, 4abc, } \\
5 \mathrm{abc}, 6 \mathrm{abc}\end{array}$ & $2 \mathrm{~b}, 3 \mathrm{ac}, 4 \mathrm{~b}, 5 \mathrm{~b}, 6 \mathrm{~b}$ & Krammer \& Lange-Bertalot (1991a) \\
\hline $\begin{array}{l}\text { A. ambigua var. ambigua f. spiralis (Skuja) } \\
\text { Ludwig \& Valente-Moreira (Figure 10) }\end{array}$ & SH:10.9; D:3.9; S:14 & $3 \mathrm{c}$ & & Ludwig \& Valente-Moreira (1990) \\
\hline $\begin{array}{l}\text { A. brasiliensis Tremarin, Torgan \& T. Ludwig } \\
\text { (Figure 11) }\end{array}$ & $\begin{array}{l}\text { SH:3.8-6.5; D:8.4-9.8; } \\
\text { S:13-14; A:6-8 }\end{array}$ & $\begin{array}{l}1 \mathrm{~b}, 2 \mathrm{abc}, 3 \mathrm{ac}, 5 \mathrm{a}, 6 \mathrm{a}, \\
6 \mathrm{~b}\end{array}$ & $1 \mathrm{~b}$ & Tremarin et al. (2012) \\
\hline $\begin{array}{l}\text { A. granulata var. angustissima (O. Müller) } \\
\text { Simonsen (Figures 17-18) }\end{array}$ & $\begin{array}{c}\text { SH:11.5-14.8; D:2.7-2.8; } \\
\text { S:3; A:14-15 }\end{array}$ & $\begin{array}{c}2 \mathrm{abc}, 3 \mathrm{ab}, 4 \mathrm{ac}, 5 \mathrm{a} \\
6 \mathrm{abc}\end{array}$ & $4 c, 6 c$ & Krammer \& Lange-Bertalot (1991a) \\
\hline $\begin{array}{l}\text { A. granulata (Ehrenberg) Simonsen var. } \\
\text { granulata (Figure 25) }\end{array}$ & $\begin{array}{l}\text { SH:13.7-13.8; D:5.1-5.2; } \\
\text { S:5-6; A:16-17 }\end{array}$ & $\begin{array}{l}\text { 2abc, 3abc, } 4 \mathrm{abc}, 5 \mathrm{abc}, \\
6 \mathrm{abc}\end{array}$ & $2 \mathrm{c}, 3 \mathrm{ab}, 4 \mathrm{c}, 5 \mathrm{~b}, 6 \mathrm{abc}$ & Krammer \& Lange-Bertalot (1991a) \\
\hline $\begin{array}{l}\text { A. herzogii (Lemmermann) Simonsen var. } \\
\text { herzogii (Figures 12-14) }\end{array}$ & SH: 3.7-7.1; D: 3.3-5.9 & $2 \mathrm{bc}, 4 \mathrm{~b}, 5 \mathrm{~b}, 6 \mathrm{bc}$ & & Hickel \& Häkansson (1991) \\
\hline A. tenella (Nygaard) Simonsen (Figures 8-9) & SH:0.7-1.7; D: 5.6-6 & $\begin{array}{l}1 \mathrm{bc}, 2 \mathrm{bc}, 3 \mathrm{abc}, 4 \mathrm{abc} \\
5 \mathrm{abc}, 6 \mathrm{c}\end{array}$ & $2 \mathrm{a}, 3 \mathrm{abc}, 5 \mathrm{bc}, 6 \mathrm{ac}$ & $\begin{array}{l}\text { Siver \& Kling (1997), } \\
\text { Camburn \& Charles (2000) }\end{array}$ \\
\hline Aulacoseira sp. (Figures 5-7) & $\mathrm{SH}: 1-1.8 ; \mathrm{D}: 4.5-4.9$ & $2 \mathrm{bc}, 3 \mathrm{ab}, 4 \mathrm{ac}, 5 \mathrm{abc}$ & $6 \mathrm{c}$ & \\
\hline \multicolumn{5}{|l|}{ Fragilariaceae } \\
\hline Fragilaria mesolepta Rabenhorst (Figure 19) & L:21.8; W:2.8; S:16 & & $5 \mathrm{a}$ & Tuji \& Williams (2008b) \\
\hline F. crotonensis Kitton (Figures 31-34) & L:60.8-76; W:2-2.6; S:17 & $\begin{array}{c}4 \mathrm{c}, 6 \mathrm{~b}, 2 \mathrm{abc}, 3 \mathrm{~b}, 4 \mathrm{ab} \\
5 \mathrm{abc}, 6 \mathrm{abc}\end{array}$ & $1 \mathrm{ac}$ & Patrick \& Reimer (1966) \\
\hline F. gracilis Ostrup (Figures 26-28) & $\mathrm{L}: 40.2 ; \mathrm{W}: 2.2 ; \mathrm{S}: 22$ & $4 \mathrm{~b}$ & $2 \mathrm{c}, 3 \mathrm{a}, 4 \mathrm{bc}, 5 \mathrm{c}, 6 \mathrm{ac}$ & Krammer \& Lange-Bertalot (1991a) \\
\hline $\begin{array}{l}\text { F. parva (Grunow) Tuji \& Williams } \\
\text { (Figures } 40-43 \text { ) }\end{array}$ & $\begin{array}{l}\mathrm{L}: 26.6-51.6 ; \mathrm{W}: 2.5-2.6 \\
\mathrm{~S}: 15-16\end{array}$ & $\begin{array}{l}1 \mathrm{~b}, 2 \mathrm{abc}, 3 \mathrm{ab}, 4 \mathrm{ac} \\
6 \mathrm{abc}\end{array}$ & $\begin{array}{c}1 \mathrm{~b}, 2 \mathrm{abc} \\
3 \mathrm{~b}, 4 \mathrm{c}, 5 \mathrm{abc}, 6 \mathrm{abc}\end{array}$ & Tuji \& Williams (2008c) \\
\hline $\begin{array}{l}\text { F. pectinallis (Müll) Lyngbye (Figures 21- } \\
24,220-222 \text { ) }\end{array}$ & $\begin{array}{c}\mathrm{L}: 20.8-28.0 ; \mathrm{W}: 3.5-3.1 \\
\mathrm{~S}: 16-18\end{array}$ & $\begin{array}{l}\text { 1a, 2ac, 3abc, 4abc, } \\
5 \mathrm{a}, 6 \mathrm{abc}\end{array}$ & $1 \mathrm{c}, 2 \mathrm{~b}, 4 \mathrm{bc}, 5 \mathrm{bc}, 6 \mathrm{abc}$ & $\begin{array}{l}\text { Tuji \& Williams (2008a) as } F \text {. } \\
\text { capitellata (Grun.) Pet., Wetzel \& } \\
\text { Ector (2015) }\end{array}$ \\
\hline $\begin{array}{l}\text { F. tenera (W. Smith) Lange-Bertalot } \\
\text { (Figures 35-37) }\end{array}$ & $\begin{array}{l}\mathrm{L}: 34.6-49.4 ; \mathrm{W}: 1.9-3.0 \\
\mathrm{~S}: 20\end{array}$ & $\begin{array}{c}1 \mathrm{abc}, 2 \mathrm{c}, 3 \mathrm{abc}, 4 \mathrm{abc} \\
5 \mathrm{abc}, 6 \mathrm{abc}\end{array}$ & $\begin{array}{c}1 \mathrm{a}, 2 \mathrm{ab} \\
3 \mathrm{~b}, 4 \mathrm{bc}, 5 \mathrm{~b}, 6 \mathrm{a}\end{array}$ & Zelazna-Wieczorek (2011) \\
\hline $\begin{array}{l}\text { F. microvaucheriae Wetzel \& Ector } \\
\text { (Figures 29-30) }\end{array}$ & $\begin{array}{c}\mathrm{L}: 10.7-11.4 ; \mathrm{W}: 4.1-4.4 \\
\mathrm{~S}: 9-10\end{array}$ & $2 \mathrm{abc}, 3 \mathrm{~b}, 4 \mathrm{abc}, 6 \mathrm{~b}$ & $2 \mathrm{abc}, 3 \mathrm{c}, 4 \mathrm{bc}, 6 \mathrm{ab}$ & Tuji \& Williams (2006) \\
\hline $\begin{array}{l}\text { Fragilariforma javanica (Hustedt) Wetzel, } \\
\text { Morales \& Ector (Figure 20) }\end{array}$ & $\begin{array}{c}\text { L:27.9-69.8; W:5.5-6.1; } \\
\text { S: } 19\end{array}$ & $2 \mathrm{c}$ & $2 b$ & Wetzel et al. (2013a) \\
\hline Ulnaria acus (Kützing) Aboal (Figure 38) & $\begin{array}{c}\mathrm{L}: 114.9-140.7 \\
\mathrm{~W}: 3.2-3.4 ; \mathrm{S}: 16\end{array}$ & & $1 \mathrm{a}$ & Levkov et al. (2007) \\
\hline U. ulna (Nitzsch) Compère (Figure 39) & L:91.2; W:4.9; S:11 & $1 \mathrm{~b}, 2 \mathrm{~b}, 3 \mathrm{~b}, 4 \mathrm{c}, 5 \mathrm{a}, 6 \mathrm{ac}$ & $2 \mathrm{abc}, 6 \mathrm{ab}$ & Levkov et al. (2007) \\
\hline \multicolumn{5}{|l|}{ Eunotiaceae } \\
\hline $\begin{array}{l}\text { Actinella leontopithecus-rosalia Costa } \\
\text { (Figure 52) }\end{array}$ & $\mathrm{L}: 25.1 ; \mathrm{W}: 3.2 ; \mathrm{S}: 18$ & & $1 \mathrm{c}, 5 \mathrm{~b}$ & Costa (1995), Tremarin et al. (2016) \\
\hline
\end{tabular}


Marra R.C. et al.

Table 3. Continued..

\begin{tabular}{|c|c|c|c|c|}
\hline \multirow{2}{*}{ Species Name (Figure) } & \multirow{2}{*}{$\begin{array}{l}\text { Metric }(\mu \mathrm{m}) \& \text { meristic } \\
\quad(\text { in } 10 \mu \mathrm{m}) \text { limits }\end{array}$} & \multicolumn{2}{|c|}{ Month of occurrence at sampling sites } & \multirow{2}{*}{ Consulted literature } \\
\hline & & June & November & \\
\hline $\begin{array}{l}\text { Desmogonium ossiculum Metzeltin \& Lange- } \\
\text { Bertalot (Figure } 73 \text { ) }\end{array}$ & $\begin{array}{l}\text { L:114.1-186.9; W:8.7; } \\
\text { S:14-15 }\end{array}$ & $2 \mathrm{c}, 3 \mathrm{a}$ & $2 \mathrm{a}$ & Metzeltin \& Lange Bertalot (2007) \\
\hline $\begin{array}{l}\text { D. transfugum (Metzeltin \& Lange-Bertalot) } \\
\text { Metzeltin \& Lange-Bertalot (Figure 74) }\end{array}$ & $\begin{array}{l}\text { L:139.5-198.7; } \\
\text { W:4.4-6.1; } \\
\text { S:14-16 }\end{array}$ & 1a & & Metzeltin \& Lange-Bertalot (1998,) \\
\hline $\begin{array}{l}\text { Eunotia bilunaris (Ehrenberg) Mills } \\
\text { (Figure } 48 \text { ) }\end{array}$ & $\begin{array}{l}\text { L:22-43.7; W:3.1-3.4; } \\
\text { S: } 17\end{array}$ & $\begin{array}{c}1 \mathrm{abc}, 2 \mathrm{bc}, 3 \mathrm{a}, 4 \mathrm{a}, 5 \mathrm{a} \\
6 \mathrm{bc}\end{array}$ & $\begin{array}{l}1 \mathrm{ab}, 2 \mathrm{ab}, 3 \mathrm{a}, 4 \mathrm{ac} \\
5 \mathrm{bc}, 6 \mathrm{c}\end{array}$ & Lange-Bertalot \& Metzeltin (1996) \\
\hline E. camelus Ehrenberg (Figures 54-55) & $\begin{array}{c}\mathrm{L}: 23.2-44.2 ; \mathrm{W}: 5.4-8.5 \\
\mathrm{~S}: 11-14\end{array}$ & $1 \mathrm{ab}, 2 \mathrm{a}$ & $1 b c, 5 b$ & $\begin{array}{l}\text { Reichardt (1995), Metzeltin et al. } \\
\text { (2005) }\end{array}$ \\
\hline $\begin{array}{l}\text { Eunotia cf. formicina Lange-Bertalot } \\
\text { (Figure 53) }\end{array}$ & $\begin{array}{l}\mathrm{L}: 46.2-49.8 ; \mathrm{W}: 6-6.4 \\
\mathrm{~S}: 13-15\end{array}$ & $2 \mathrm{c}$ & & Lange-Bertalot et al. (2011) \\
\hline $\begin{array}{l}\text { E. desmogonioides Metzeltin \& Lange-Bertalot } \\
\text { (Figure } 72 \text { ) }\end{array}$ & $\begin{array}{l}\text { L:109.3-125.5; } \\
\text { W:5.8-6.7; S:15 }\end{array}$ & $1 \mathrm{a}, 3 \mathrm{ab}, 5 \mathrm{ab}$ & $2 \mathrm{~b}$ & Metzeltin \& Lange-Bertalot (2002) \\
\hline $\begin{array}{l}\text { E. intermedia (Krasske) Nörpel-Schempp \& } \\
\text { Lange-Bertalot (Figures 49-50) }\end{array}$ & $\begin{array}{l}\text { L:9.7-17.8; W:2.9-3.1; } \\
\text { S:14-15 }\end{array}$ & $\begin{array}{l}1 \mathrm{ac}, 2 \mathrm{abc}, 3 \mathrm{bc}, 4 \mathrm{ab} \\
5 \mathrm{abc}, 6 \mathrm{abc}\end{array}$ & $\begin{array}{c}1 \mathrm{abc}, 2 \mathrm{abc} \\
3 \mathrm{abc}, 5 \mathrm{~b}, 6 \mathrm{bc}\end{array}$ & Lange-Bertalot et al. (2011) \\
\hline E. luna var. trapezica Hustedt (Figure 59) & L:28.7; W:11; S:12 & $6 \mathrm{~b}$ & & Frenguelli (1941), Simonsen (1987) \\
\hline $\begin{array}{l}\text { E. meridiana Metzeltin \& Lange-Bertalot } \\
\text { (Figure 56-58) }\end{array}$ & $\begin{array}{l}\mathrm{L}: 17.2-26.9 ; \mathrm{W}: 4-5 \\
\mathrm{~S}: 12-13\end{array}$ & $2 a, 4 b, 6 b$ & $\begin{array}{c}1 \mathrm{abc} \\
3 \mathrm{ab}, 4 \mathrm{~b}, 5 \mathrm{c}, 6 \mathrm{bc}\end{array}$ & Metzeltin \& Lange-Bertalot (1998) \\
\hline E. monodon Ehrenberg (Figure 45) & $\mathrm{L}: 69.2 ; \mathrm{W}: 9.5 ; \mathrm{S}: 10$ & $4 \mathrm{a}$ & & Hofmann et al. (2013) \\
\hline E. minor (Kützing) Grunow (Figure 44) & $\begin{array}{l}\text { L: } 19.9-31.8 ; \mathrm{W}: 4.1-5.1 \\
\text { S: } 14-17\end{array}$ & $3 \mathrm{c}, 6 \mathrm{~b}$ & $2 \mathrm{ab}, 3 \mathrm{ab}, 4 \mathrm{bc}, 6 \mathrm{a}$ & Lange-Bertalot et al. (2011) \\
\hline E. naegelii Migulla (Figures 46-47) & $\begin{array}{l}\text { L:61.1-106.6; W:2.5-2.9; } \\
\text { S:16-20 }\end{array}$ & $1 \mathrm{ab}, 3 \mathrm{c}$ & $3 a$ & Krammer \& Lange-Bertalot (1991a) \\
\hline E. neocompacta Mayama (Figure 51) & L:18.4; W:2.7; S:20 & & $6 \mathrm{a}$ & Lange-Bertalot et al. (2011) \\
\hline $\begin{array}{l}\text { E. paratridentula Lange-Bertalot \& } \\
\text { Kulikovskiy (Figure } 61 \text { ) }\end{array}$ & $\begin{array}{l}\mathrm{L}: 13.6-15.4 ; \mathrm{W}: 3-3.3 \\
\mathrm{~S}: 22\end{array}$ & $2 \mathrm{abc}$ & & Kulikovskiy et al. (2010) \\
\hline $\begin{array}{l}\text { E. pseudosudetica Metzeltin, Lange-Bertalot \& } \\
\text { García-Rodríguez (Figure 63-64) }\end{array}$ & $\begin{array}{l}\mathrm{L}: 29.1-59.9 ; \mathrm{W}: 4.1-5.2 \\
\mathrm{~S}: 12\end{array}$ & $\begin{array}{l}\text { 1abc, } 2 a b c, 3 b, 4 a b \\
5 a b c, 6 a b c\end{array}$ & $\begin{array}{l}1 \mathrm{abc}, 2 \mathrm{abc}, 3 \mathrm{abc}, 4 \mathrm{abc} \\
5 \mathrm{~b}, 6 \mathrm{abc}\end{array}$ & Metzeltin et al. (2005) \\
\hline $\begin{array}{l}\text { E. pyramidata Hustedt var. pyramidata } \\
\text { (Figure } 60 \text { ) }\end{array}$ & $\begin{array}{l}\text { L:19.5-33.4; W:5.9-8.8; } \\
\text { S:13-15 }\end{array}$ & & $5 b$ & Frenguelli (1941) \\
\hline $\begin{array}{l}\text { E. rabenhorstii var. monodon Cleve \& Grunow } \\
\text { (Figure } 62 \text { ) }\end{array}$ & L:17.6; W:6.5; S:13 & $1 \mathrm{a}$ & & Patrick \& Reimer (1966) \\
\hline $\begin{array}{l}\text { E. yanomami Metzeltin \& Lange-Bertalot } \\
\text { (Figures } 65-67 \text { ) }\end{array}$ & $\begin{array}{l}\text { L: }-89-152.7 \\
\text { W: } 17.2-18.7 \\
\quad \text { S: } 10-13\end{array}$ & $6 \mathrm{~b}, \mathrm{c}$ & & Metzeltin \& Lange-Bertalot (1998) \\
\hline \multicolumn{5}{|l|}{ Cymbellaceae } \\
\hline $\begin{array}{l}\text { Cymbella aspera (Ehrenberg) Cleve } \\
\text { (Figure 91) }\end{array}$ & $\begin{array}{c}\text { L:108.4-158.3; } \\
\text { W:20.6-25.2; S:9-10; } \\
\text { A:10-11 }\end{array}$ & $2 \mathrm{c}, 5 \mathrm{bc}, 6 \mathrm{ac}$ & $5 \mathrm{a}$ & $\begin{array}{l}\text { Patrick \& Reimer (1975), Krammer } \\
\text { (2002) }\end{array}$ \\
\hline $\begin{array}{l}\text { Cymbopleura naviculiformis var. } \\
\text { naviculiformis (Auerswald) Krammer } \\
\text { (Figure 71) }\end{array}$ & $\begin{array}{c}\mathrm{L}: 27.5-31.9 ; \mathrm{W}: 7.1-7.9 \\
\mathrm{~S}: 14\end{array}$ & $2 \mathrm{bc}, 4 \mathrm{c}, 5 \mathrm{bc}, 6 \mathrm{abc}$ & $5 \mathrm{~b}, 6 \mathrm{c}$ & Krammer (2003) \\
\hline Encyonema incurvatum Krammer (Figures 68-70) & $\begin{array}{l}\mathrm{L}: 27.2-32.2 ; \mathrm{W}: 7.5-8.1 \\
\mathrm{~S}: 10-11\end{array}$ & 1a, 2abc, 3a, 4abc, 6ab & $5 b, 6 c$ & Krammer (1997a) \\
\hline E. neogracile Krammer (Figure 80) & $\begin{array}{c}\mathrm{L}: 26.4-47.4 ; \mathrm{W}: 5.5-7.6 \\
\text { S:12-14 }\end{array}$ & $\begin{array}{c}1 \mathrm{abc}, 2 \mathrm{abc}, 3 \mathrm{bc}, 4 \mathrm{bc} \\
5 \mathrm{abc}, 6 \mathrm{abc}\end{array}$ & $1 \mathrm{c}, 2 \mathrm{abc}, 3 \mathrm{~b}, 4 \mathrm{c}, 5 \mathrm{~b}, 6 \mathrm{c}$ & Krammer (1997a) \\
\hline E. silesiacum (Bleisch) Mann (Figures 75-77) & $\begin{array}{l}\mathrm{L}: 20.5-24.7 ; \mathrm{W}: 6.5-8.1 ; \\
\mathrm{S}: 10-13\end{array}$ & $2 \mathrm{a}, 6 \mathrm{~b}$ & $2 b$ & Krammer (1997a) \\
\hline E. vulgare var. vulgare Krammer (Figure 78 ) & L:31.4; W:8.6; S:10 & $4 \mathrm{c}$ & & Krammer (1997a) \\
\hline $\begin{array}{l}\text { Encyonopsis frequentiformis Metzeltin \& } \\
\text { Krammer (Figure 79) }\end{array}$ & $\begin{array}{l}\text { L:38.4-39.7; W:9.3-9.5; } \\
\text { S:15-16 }\end{array}$ & & $3 a, 3 b, 4 b, 5 b$ & Metzeltin \& Lange-Bertalot (1998) \\
\hline $\begin{array}{l}\text { E. microcephala (Grunow) Krammer } \\
\text { (Figures 95-96) }\end{array}$ & $\begin{array}{l}\text { L:17.6-23.1; W:4.0; } \\
\text { S:23-24 }\end{array}$ & $\begin{array}{c}2 \mathrm{abc}, 3 \mathrm{ab}, 4 \mathrm{c}, 5 \mathrm{bc}, \\
6 \mathrm{abc}\end{array}$ & $3 a, 5 b$ & Krammer (1997b) \\
\hline $\begin{array}{l}\text { Geissleria lateropunctata (Wallace) Potapova } \\
\& \text { Winter (Figure 89) }\end{array}$ & $\begin{array}{l}\mathrm{L}: 20.5-21.8 ; \mathrm{W}: 7.1-7.7 \\
\text { S: } 18-19\end{array}$ & $2 \mathrm{a}, 4 \mathrm{c}, 5 \mathrm{~b}, 6 \mathrm{c}$ & $6 \mathrm{~b}$ & Kulikovskiy et al. (2014) \\
\hline $\begin{array}{l}\text { G. punctifera (Hustedt) Metzeltin, Lange- } \\
\text { Bertalot \& García-Rodríguez (Figure } 88 \text { ) }\end{array}$ & $\begin{array}{c}\mathrm{L}: 20.8-25.5 ; \mathrm{W}: 6.3-6.6 \\
\mathrm{~S}: 17\end{array}$ & $2 b, 6 a$ & & $\begin{array}{l}\text { Kulikovskiy et al. (2014), Torgan \& } \\
\text { Oliveira (2001) }\end{array}$ \\
\hline $\begin{array}{l}\text { Placoneis elginensis (Gregory) Cox } \\
\text { (Figures } 81-82 \text { ) }\end{array}$ & $\begin{array}{l}\text { L:30.2-34.8; W:9.9-10.1; } \\
\text { S:13 }\end{array}$ & $4 \mathrm{a}$ & & $\begin{array}{l}\text { Hustedt (1961-1966), Hofmann et al. } \\
\text { (2013) }\end{array}$ \\
\hline
\end{tabular}


Table 3. Continued...

\begin{tabular}{|c|c|c|c|c|}
\hline \multirow{2}{*}{ Species Name (Figure) } & \multirow{2}{*}{$\begin{array}{l}\text { Metric }(\mu \mathrm{m}) \& \text { meristic } \\
\quad \text { (in } 10 \mu \mathrm{m}) \text { limits }\end{array}$} & \multicolumn{2}{|c|}{ Month of occurrence at sampling sites } & \multirow{2}{*}{ Consulted literature } \\
\hline & & June & November & \\
\hline $\begin{array}{l}\text { Placoneis symmetrica (Hustedt) } \\
\text { Lange-Bertalot (Figure } 83 \text { ) }\end{array}$ & L:31.8; W:9.6; S:12 & $4 \mathrm{~b}$ & & Cox (2003), Hofmann et al. (2013) \\
\hline \multicolumn{5}{|l|}{ Gomphonemataceae } \\
\hline $\begin{array}{l}\text { Gomphonema guaraniarum Metzeltin \& } \\
\text { Lange-Bertalot (Figures } 84-87 \text { ) }\end{array}$ & $\begin{array}{c}\text { L:68.6-54.7; } \\
\text { W:11.1-11.5; S:10-11 }\end{array}$ & $\begin{array}{l}1 \mathrm{abc}, 2 \mathrm{ab}, 3 \mathrm{ac}, 4 \mathrm{bc} \\
5 \mathrm{bc}, 6 \mathrm{abc}\end{array}$ & $1 \mathrm{ab}, 2 \mathrm{ab}, 3 \mathrm{ac}, 4 \mathrm{bc}, 5 \mathrm{bc}$ & Metzeltin \& Lange-Bertalot (2007) \\
\hline G. hawaiiensis Reichardt (Figure 103) & $\mathrm{L}: 35.2 ; \mathrm{W}: 7.3 ; \mathrm{S}: 16$ & $6 \mathrm{~b}$ & & Reichardt (2005) \\
\hline G. lagenula Kützing (Figure 104) & $\begin{array}{l}\text { L:15.6-21.1; W:5.4-6.1; } \\
\text { S:16 }\end{array}$ & $\begin{array}{l}\text { 1abc, } 2 \mathrm{abc}, 3 \mathrm{abc}, 4 \mathrm{abc} \\
5 \mathrm{abc}, 6 \mathrm{ab}\end{array}$ & $1 \mathrm{a}, \mathrm{c}, 3 \mathrm{ab}, 6 \mathrm{c}$ & Krammer \& Lange-Bertalot (1991b) \\
\hline $\begin{array}{l}\text { G. graciloides Hustedt } \\
\text { (Figures } 97-101,214-216 \text { ) }\end{array}$ & $\begin{array}{l}\mathrm{L}: 25.4-47.9 ; \mathrm{W}: 7.8-8.5 \\
\mathrm{~S}: 11-14\end{array}$ & $1 \mathrm{c}$ & & Reichardt (2015) \\
\hline $\begin{array}{l}\text { G. naviculoides W. Smith } \\
\text { (Figures } 116-117,213 \text { ) }\end{array}$ & $\begin{array}{l}\mathrm{L}: 29.1-56.3 ; \mathrm{W}: 6.6-9.3 \\
\mathrm{~S}: 14-16\end{array}$ & $\begin{array}{l}1 \mathrm{abc}, 2 \mathrm{ab}, 3 \mathrm{ac}, 4 \mathrm{bc} \\
5 \mathrm{bc}, 6 \mathrm{abc}\end{array}$ & $2 b, 3 a, 4 c, 5 b, 6 b c$ & Reichardt (2015) \\
\hline $\begin{array}{l}\text { G. parvulum (Kützing) Van Heurck var. } \\
\text { parvulum (Figure 121-122) }\end{array}$ & $\begin{array}{l}\text { L:13.9-17.6; W:3.9-4.4; } \\
\text { S:15 }\end{array}$ & $1 \mathrm{bc}, 2 \mathrm{a}, 3 \mathrm{a}, 5 \mathrm{a}$ & $1 b c, 3 a, 6 c$ & Krammer \& Lange-Bertalot (1991b) \\
\hline $\begin{array}{l}\text { G. parvulum f. saprophilum Lange-Bertalot \& } \\
\text { Reichardt (Figure 120) }\end{array}$ & $\begin{array}{l}\text { L:11.8-16.4; W:4.6-6.4; } \\
\text { S:17 }\end{array}$ & $\begin{array}{l}1 \mathrm{abc}, 2 \mathrm{ab}, 3 \mathrm{ac}, 4 \mathrm{bc} \\
5 \mathrm{bc}, 6 \mathrm{a}\end{array}$ & $1 \mathrm{abc}, 3 \mathrm{ac}, 5 \mathrm{bc}, 6 \mathrm{a}$ & Krammer \& Lange-Bertalot (1991b) \\
\hline $\begin{array}{l}\text { G. parvulum var. subcapitata Grunow } \\
\text { (Figure } 90 \text { ) }\end{array}$ & $\begin{array}{l}\mathrm{L}: 12.7-26.1 ; \mathrm{W}: 3.7-4.8 \\
\mathrm{~S}: 12-15\end{array}$ & $1 \mathrm{abc}, 2 \mathrm{ab}$ & $1 \mathrm{abc}$ & $\begin{array}{l}\text { Van Heurck (1880), Krammer \& } \\
\text { Lange-Bertalot (1986) }\end{array}$ \\
\hline $\begin{array}{l}\text { G. pseudoaugur Lange-Bertalot } \\
\text { (Figures } 92-94 \text { ) }\end{array}$ & $\begin{array}{l}\mathrm{L}: 27.5-31.8 ; \mathrm{W}: 7.2-8.3 \\
\text { S: } 15\end{array}$ & $3 \mathrm{a}$ & $6 \mathrm{c}$ & Krammer \& Lange-Bertalot (1986) \\
\hline G. subtile Ehrenberg (Figure 102) & L:48.2; W:7.83; S:9 & $4 \mathrm{~b}$ & & $\begin{array}{l}\text { Krammer \& Lange-Bertalot (1986) } \\
\text { Patrick \& Reimer (1975) }\end{array}$ \\
\hline Gomphonema sp. (Figures 118-119) & $\begin{array}{l}\mathrm{L}: 23.6-33.2 ; \mathrm{W}: 3.8-4.1 ; \\
\mathrm{S}: 14\end{array}$ & $1 \mathrm{abc}, 2 \mathrm{c}$ & & \\
\hline \multicolumn{5}{|l|}{ Achnanthidiaceae } \\
\hline $\begin{array}{l}\text { Achnanthidium caledonicum (Lange-Bertalot) } \\
\text { Lange-Bertalot (Figures 105-107) }\end{array}$ & L:17.4-24.8; W:2.5-2.7 & $\begin{array}{l}\text { 1abc, } 2 \mathrm{abc}, 3 \mathrm{abc}, 4 \mathrm{ac} \\
5 \mathrm{abc}, 6 \mathrm{abc}\end{array}$ & $\begin{array}{c}1 \mathrm{abc}, 2 \mathrm{~b}, 3 \mathrm{ab}, 4 \mathrm{bc}, 5 \mathrm{~b}, \\
6 \mathrm{ab}\end{array}$ & Wojtal et al. (2011) \\
\hline $\begin{array}{l}\text { A. catenatum (Bily \& Marvan) Lange-Bertalot } \\
\text { (Figures } 110-112 \text { ) }\end{array}$ & L: 13.6-18.7; W:2.9 & $\begin{array}{l}\text { 1abc, 2ab, 3abc, 4abc, } \\
5 \mathrm{abc}, 6 \mathrm{abc}\end{array}$ & $1 b, 2 b, 3 a, 5 b, 6 c$ & Krammer \& Lange-Bertalot (1991b) \\
\hline $\begin{array}{l}\text { A. eutrophilum (Lange-Bertalot) Lange- } \\
\text { Bertalot (Figures 125-126) }\end{array}$ & L:8.3-10.7; W:3-3.1 & $\begin{array}{c}1 \mathrm{ab}, 2 \mathrm{bc}, 3 \mathrm{ab}, 4 \mathrm{ac} \\
5 \mathrm{bc}, 6 \mathrm{a}\end{array}$ & $1 \mathrm{abc}, 5 \mathrm{a}$ & Hlúbiková et al. (2011) \\
\hline $\begin{array}{l}\text { A. exiguum (Grunow) Czarnecki } \\
\text { (Figures 123-124) }\end{array}$ & L:10.6-13.8; W:4.4-4.9 & $4 \mathrm{c}$ & & Krammer \& Lange-Bertalot (1991b) \\
\hline $\begin{array}{l}\text { A. macrocephalum (Hustedt) Round \& } \\
\text { Bukhtiyarova (Figures 114-115, 219) }\end{array}$ & L:9.1-11.6; W:2.6-2.7 & $\begin{array}{l}\text { 1ab, 2abc, 3abc, 4abc, } \\
5 \mathrm{abc}, 6 \mathrm{abc}\end{array}$ & $1 \mathrm{abc}, 2 \mathrm{~b}, 3 \mathrm{bc}, 5 \mathrm{~b}, 6 \mathrm{bc}$ & $\begin{array}{l}\text { Ponader \& Potapova (2007), } \\
\text { Taylor et al. (2007) }\end{array}$ \\
\hline $\begin{array}{l}\text { A. minutissimum (Kützing) Czarnecki } \\
\text { (Figure 113) }\end{array}$ & L:7.9-16.4; W:2.5-3.1 & $\begin{array}{c}\text { 1abc, } 2 \mathrm{abc}, 3 \mathrm{abc}, 4 \mathrm{abc} \\
5 \mathrm{abc}, 6 \mathrm{bc}\end{array}$ & $\begin{array}{l}1 \mathrm{abc}, 2 \mathrm{~b}, 3 \mathrm{abc}, 4 \mathrm{abc} \\
5 \mathrm{ab}, 6 \mathrm{abc}\end{array}$ & $\begin{array}{l}\text { Potapova (2009), Siver \& Hamilton } \\
\text { (2011) }\end{array}$ \\
\hline $\begin{array}{l}\text { Lemnicola hungarica (Grunow) Round \& } \\
\text { Basson (Figure 129) }\end{array}$ & L:23.1; W:7.1; S:20 & & $1 \mathrm{~b}$ & Round \& Basson (1997) \\
\hline $\begin{array}{l}\text { Planothidium biporomum (Hohn et Hellerman) } \\
\text { Lange-Bertalot (Figure 130) }\end{array}$ & L:21.7; W:6.8; S:13 & $2 b$ & & Wetzel et al. (2013b) \\
\hline $\begin{array}{l}\text { P. heteromorphum (Grunow) Lange-Bertalot } \\
\text { (Figures 131-132) }\end{array}$ & L:34.5; W:13.1; S:10 & $4 \mathrm{~b}$ & & Metzeltin \& Lange-Bertalot (1998) \\
\hline $\begin{array}{l}\text { P. rostratum (Oestrup) Lange-Bertalot } \\
\text { (Figures 108-109) }\end{array}$ & L:12.6; W:5; S:14 & $3 a$ & & $\begin{array}{l}\text { Krammer \& Lange-Bertalot (1991b), } \\
\text { Levkov et al. (2007) }\end{array}$ \\
\hline \multicolumn{5}{|l|}{ Diadesmidaceae } \\
\hline $\begin{array}{l}\text { Humidophila contenta (Grunow) Lowe et al. } \\
\text { (Figure 128) }\end{array}$ & $\mathrm{L}: 7.5 ; \mathrm{W}: 2.2$ & $1 \mathrm{a}, 2 \mathrm{a}, 2 \mathrm{~b}$ & & $\begin{array}{l}\text { Metzeltin \& Lange-Bertalot (2007), } \\
\text { Lowe et al. (2014) }\end{array}$ \\
\hline $\begin{array}{l}\text { H. implicata (Moser, Lange-Bertalot \& } \\
\text { Metzeltin) Lowe et al. (Figure 127) }\end{array}$ & $\mathrm{L}: 10.4 ; \mathrm{W}: 2.9$ & $5 \mathrm{a}$ & & Lowe et al. (2014) \\
\hline \multicolumn{5}{|l|}{ Amphipleuraceae } \\
\hline $\begin{array}{l}\text { Frustulia acidophilissima Wydrzycka \& } \\
\text { Lange-Bertalot (Figure 156) }\end{array}$ & L:31.8; W:10.8 & $2 \mathrm{a}, 4 \mathrm{~b}$ & $6 a$ & Metzeltin \& Lange-Bertalot (2007) \\
\hline $\begin{array}{l}\text { F. crassinervia (Brébisson) Costa } \\
\text { (Figures 133-134) }\end{array}$ & L:32.7-34.2; W:7.9-8.8 & $1 \mathrm{ab}, 2 \mathrm{abc} ; 6 \mathrm{ab}$ & $5 \mathrm{~b}$ & Metzeltin \& Lange-Bertalot (1998) \\
\hline $\begin{array}{l}\text { F. guayanensis Metzeltin \& Lange Bertalot } \\
\text { (Figure 137) }\end{array}$ & L:34.5-34.4; W:7.8-7.6 & $2 \mathrm{a}, 6 \mathrm{c}$ & & Metzeltin \& Lange-Bertalot (1998) \\
\hline F. quadrisinuata Lange-Bertalot (Figure 157) & L:58.9; W:14.5 & $6 \mathrm{~b}$ & & Metzeltin \& Lange-Bertalot (1998) \\
\hline $\begin{array}{l}\text { F. undosa Metzeltin \& Lange-Beratlot } \\
\text { (Figures 135-136) }\end{array}$ & L:32.7-39.1;W:8.4-9.2 & $2 a b c, 6 b$ & & $\begin{array}{l}\text { Metzeltin \& Lange-Bertalot (1998) } \\
\text { Metzeltin \& Lange-Bertalot (2007) }\end{array}$ \\
\hline
\end{tabular}


Table 3. Continued...

\begin{tabular}{|c|c|c|c|c|}
\hline \multirow{2}{*}{ Species Name (Figure) } & \multirow{2}{*}{$\begin{array}{l}\text { Metric }(\mu \mathrm{m}) \& \text { meristic } \\
\quad(\text { in } 10 \mu \mathrm{m}) \text { limits }\end{array}$} & \multicolumn{2}{|c|}{ Month of occurrence at sampling sites } & \multirow{2}{*}{ Consulted literature } \\
\hline & & June & November & \\
\hline \multicolumn{5}{|l|}{ Brachysiraceae } \\
\hline Brachysira brebissonii Ross (Figure 140) & L:16.9-24.3;W:4.5-6.9 & $1 \mathrm{abc}, 2 \mathrm{ac}$ & $2 b$ & Lange-Bertalot \& Moser (1994) \\
\hline B. neoexilis Lange-Bertalot (Figure 141) & L:26.3-13.9; W:5.4-4.3 & $\begin{array}{l}\text { 1abc, 2abc, 3bc, 4abc, } \\
5 \mathrm{abc}, 6 \mathrm{abc}\end{array}$ & $\begin{array}{c}1 \mathrm{ab}, 2 \mathrm{abc}, 3 \mathrm{abc}, 4 \mathrm{c} \\
5 \mathrm{ab}, 6 \mathrm{ab}\end{array}$ & Lange-Bertalot \& Moser (1994) \\
\hline \multicolumn{5}{|l|}{ Neidiaceae } \\
\hline Neidium affine (Ehrenberg) Pfitzer (Figure 139) & L: $43.3 ; \mathrm{W}: 10.7 ; \mathrm{S}: 24$ & $6 a$ & & $\begin{array}{l}\text { Patrick \& Reimer (1966), Krammer } \\
\text { \& Lange-Bertalot (1986) }\end{array}$ \\
\hline N. iridis (Ehrenberg) Cleve (Figure 138) & $\begin{array}{c}\mathrm{L}: 76.6 ; \mathrm{W}: 30.3 ; \mathrm{S}: 17 \\
\mathrm{~A}: 9\end{array}$ & $6 a$ & & Krammer \& Lange-Bertalot (1986) \\
\hline \multicolumn{5}{|l|}{ Sellaphoraceae } \\
\hline $\begin{array}{l}\text { Sellaphora nigri (De Notaris) Wetzel \& Ector } \\
\text { (Figures 151-152) }\end{array}$ & L:8.7; W:3.6 & $4 \mathrm{c}$ & & Wetzel et al. (2015) \\
\hline $\begin{array}{l}\text { S. pupula (Kützing) Mereschkowsky } \\
\text { (Figures 146-147) }\end{array}$ & $\begin{array}{c}\mathrm{L}: 21.8-25.7 ; \mathrm{W}: 6.6-7.4 \\
\mathrm{~S}: 21-25\end{array}$ & $4 \mathrm{~b}$ & & Mann et al. (2004) \\
\hline $\begin{array}{l}\text { S. sardiniensis Lange-Bertalot, Cavacini, } \\
\text { Tagliaventi \& Alfinito (Figures 149-150, 218) }\end{array}$ & $\begin{array}{l}\text { L:9.2-12.8; W:4.2-4.5; } \\
\text { S:24 }\end{array}$ & $4 \mathrm{~b}$ & & Lange-Bertalot et al. (2003) \\
\hline $\begin{array}{l}\text { S. sassiana (Metzeltin \& Lange-Bertalot) } \\
\text { Wetzel (Figures 144-145, 217) }\end{array}$ & L:15; W:4.4; S:24 & $5 \mathrm{ac}, 6 \mathrm{c}$ & & Metzeltin \& Lange-Bertalot (1998) \\
\hline $\begin{array}{l}\text { S. saugerresii (Desm.) Wetzel \& Mann } \\
\text { (Figures } 142-143 \text { ) }\end{array}$ & $\begin{array}{l}\text { L:5.4-18.8; W:3.3-5; } \\
\text { S:17-22 }\end{array}$ & $\begin{array}{l}2 \mathrm{abc}, 3 \mathrm{ab}, 4 \mathrm{bc}, 5 \mathrm{ab} \\
6 \mathrm{abc}\end{array}$ & $3 \mathrm{ab}, 4 \mathrm{a}, 6 \mathrm{bc}$ & Wetzel et al. (2015) \\
\hline $\begin{array}{l}\text { S. ventraloconfusa (Lange-Bertalot) Metzeltin } \\
\text { \& Lange-Bertalot (Figure 153) }\end{array}$ & $\begin{array}{l}\mathrm{L}: 15.5-23.3 ; \mathrm{W}: 4.6-5.8 \\
\mathrm{~S}: 22\end{array}$ & $1 \mathrm{ab}, 2 \mathrm{c}, 4 \mathrm{a}$ & & Krammer \& Lange-Bertalot (1986) \\
\hline Sellaphora sp. (Figure 148) & L:15.9; W:5.6; S:23 & & $2 \mathrm{a}$ & \\
\hline \multicolumn{5}{|l|}{ Pinnulariaceae } \\
\hline $\begin{array}{l}\text { Chamaepinnularia brasilianopsis Metzeltin \& } \\
\text { Lange-Bertalot (Figures 171-172) }\end{array}$ & L:15; W:3.9; S:23 & $4 \mathrm{~b}$ & & Metzeltin \& Lange-Bertalot (1998) \\
\hline $\begin{array}{l}\text { Chamaepinnularia mediocris (Krasske) Lange- } \\
\text { Bertalot (Figures 154-155) }\end{array}$ & L:11.0; W:3.8; S:21 & $2 \mathrm{ac}$ & & Lange-Bertalot \& Metzeltin (1996) \\
\hline $\begin{array}{l}\text { Pinnularia acrosphaeria (Brébisson) Smith } \\
\text { (Figure 158) }\end{array}$ & L:79.24; W:13.54; S:10 & $1 \mathrm{a}$ & & Krammer (2000) \\
\hline P. brauniana (Gunow) Mills (Figure 160) & L: 49.75; W: 9.6; S:11 & 1a & & Krammer (1992) \\
\hline $\begin{array}{l}\text { P. butantanum (Krasske) Meltzeltin } \\
\text { (Figure 166) }\end{array}$ & L:87.8; W:12.7; S:15 & $1 \mathrm{a}$ & & Metzeltin \& Lange-Bertalot (1998) \\
\hline $\begin{array}{l}\text { P. divergentissima var. minor Krammer } \\
\text { (Figure 168) }\end{array}$ & $\begin{array}{l}\mathrm{L}: 23.7-27.7 ; \mathrm{W}: 4.8-5.1 ; \\
\mathrm{S}: 15\end{array}$ & $6 \mathrm{~b}$ & & Krammer (2000) \\
\hline P. gibba Ehrenberg (Figures 164-165) & $\begin{array}{c}\text { L:57.5-74.2; } \\
\text { W:10.7-11.7; S:9 }\end{array}$ & $1 \mathrm{a}$ & & Krammer (2000) \\
\hline P. latarea Krammer (Figure 159) & L:61.7; W:10.1; S:10 & 1a & & Krammer (2000) \\
\hline P. latevittata Cleve (Figure 162) & L:216.1; W:33.7; S:5 & $2 \mathrm{a}$ & & Reichardt (1995) \\
\hline P. similiformis Krammer (Figure 167) & L:38.9; W:5; S:13 & & $6 \mathrm{~b}$ & Krammer (2000) \\
\hline P. subbrevistriata Krammer (Figure 170) & $\mathrm{L}: 48.4 ; \mathrm{W}: 9.2 ; \mathrm{S}: 11$ & & $6 \mathrm{~b}$ & Krammer (2000) \\
\hline P. subcapitata Gregory (Figure 169) & $\mathrm{L}: 28.9 ; \mathrm{W}: 4.8 ; \mathrm{S}: 14$ & $4 \mathrm{~b}$ & & Krammer (2000) \\
\hline $\begin{array}{l}\text { P. subgibba var. undulata Krammer } \\
\text { (Figure } 163 \text { ) }\end{array}$ & L:84.2; W:8.8: S:12 & 1a & & Krammer (1992) \\
\hline $\begin{array}{l}\text { P. stoermeri Metzeltin \& Lange-Bertalot } \\
\text { (Figure 161) }\end{array}$ & L:157.6; W:26.6; S:8 & $4 a$ & & Metzeltin \& Lange-Bertalot (2007) \\
\hline \multicolumn{5}{|l|}{ Naviculaceae } \\
\hline $\begin{array}{l}\text { Capartogramma crucicula (Grunow) Ross } \\
\text { (Figure 173) }\end{array}$ & $\mathrm{L}: 32.5 ; \mathrm{W}: 9.7 ; \mathrm{S}: 23$ & $4 \mathrm{~b}$ & & Patrick \& Reimer (1966) \\
\hline $\begin{array}{l}\text { Hippodonta capitata ssp. iberoamericana } \\
\text { Metzeltin, Lange-Bertalot \& García-Rodríguez } \\
\text { (Figure 174) }\end{array}$ & L:20.6; W:4.9; S:10 & $2 \mathrm{~b}$ & & Metzeltin et al. (2005) \\
\hline $\begin{array}{l}\text { Mayamaea permitis (Hustedt) Bruder \& } \\
\text { Medlin (Figure 187) }\end{array}$ & L:9.2; W:3.1 & $4 \mathrm{c}$ & $3 a$ & Lange-Bertalot et al. (2003) \\
\hline Navicula angusta Grunow (Figures 175-176) & $\begin{array}{l}\text { L:40.5-45.0; W:6.1-6.4; } \\
\text { S: } 12\end{array}$ & $2 \mathrm{bc}, 3 \mathrm{a}, 4 \mathrm{bc}, 6 \mathrm{abc}$ & $3 a, 4 b, 5 a b, 6 a b$ & Krammer \& Lange-Bertalot (1986) \\
\hline N. cryptotenella Lange-Bertalot (Figure 179) & $\begin{array}{l}\mathrm{L}: 23.5-26.2 ; \mathrm{W}: 5.3-5.3 ; \\
\text { S: } 16\end{array}$ & $1 \mathrm{ab}, 2 \mathrm{bc}, 4 \mathrm{c}, 5 \mathrm{abc}, 6 \mathrm{ac}$ & $5 b, 6 b c$ & Lange-Bertalot \& Metzeltin (1996) \\
\hline N. notha Wallace (Figure 178) & $\begin{array}{l}\text { L:23.7-26.6; W:4.4-4.7; } \\
\text { S:16 }\end{array}$ & $3 \mathrm{c}, 5 \mathrm{bc}$ & & Rumrich et al. (2000) \\
\hline
\end{tabular}


Table 3. Continued...

\begin{tabular}{|c|c|c|c|c|}
\hline \multirow{2}{*}{ Species Name (Figure) } & \multirow{2}{*}{$\begin{array}{l}\text { Metric }(\mu \mathrm{m}) \& \text { meristic } \\
\quad \text { (in } 10 \mu \mathrm{m}) \text { limits }\end{array}$} & \multicolumn{2}{|c|}{ Month of occurrence at sampling sites } & \multirow{2}{*}{ Consulted literature } \\
\hline & & June & November & \\
\hline N. tridentula Krasske (Figures 181-182) & $\mathrm{L}: 17.5 ; \mathrm{W}: 3.7$ & $5 b$ & & Krammer \& Lange-Bertalot (1986) \\
\hline N. veneta Kützing (Figure 180) & L:18.8; W:5.4; S:17 & $2 \mathrm{~b}$ & & Rumrich et al. (2000) \\
\hline $\begin{array}{l}\text { N. viridulacalcis Lange-Bertalot \& Rumrich } \\
\text { (Figure 177) }\end{array}$ & $\begin{array}{c}\text { L:44.2-75.6; } \\
\text { W:10.5-11.3; S:9 }\end{array}$ & $4 a b c, 5 b, 6 c$ & $3 a, 5 a$ & Rumrich et al. (2000) \\
\hline $\begin{array}{l}\text { N. ventraloconfusa var. chilensis (Krasske) } \\
\text { Lange-Bertalot (Figures 188-189) }\end{array}$ & $\begin{array}{l}\text { L:15.9-19.2; W:5.2-5.4; } \\
\text { S:21 }\end{array}$ & $4 \mathrm{a}, 5 \mathrm{~b}$ & & $\begin{array}{l}\text { Krammer \& Lange-Bertalot (1986), } \\
\text { Lange-Bertalot \& Metzeltin (1996) }\end{array}$ \\
\hline $\begin{array}{l}\text { Nupela torganie Tremarin \& Ludwig } \\
\text { (Figures 183-184) }\end{array}$ & L:10.9-13.7; W: 4.1-5 & $\begin{array}{c}1 \mathrm{ab}, 2 \mathrm{ab}, 3 \mathrm{ab}, 4 \mathrm{ab} \\
5 \mathrm{~b}, 6 \mathrm{c}\end{array}$ & $6 \mathrm{c}$ & Tremarin et al. (2015) \\
\hline \multicolumn{5}{|l|}{ Stauroneidaceae } \\
\hline $\begin{array}{l}\text { Craticula riparia (Hustedt) Lange-Bertalot } \\
\text { (Figures 192-193) }\end{array}$ & $\begin{array}{l}\mathrm{L}: 37.6-38.4 ; \mathrm{W}: 7.2-8.1 \\
\mathrm{~S}: 22\end{array}$ & $2 \mathrm{ab}, 3 \mathrm{a}, 4 \mathrm{~b}$ & & Krammer \& Lange-Bertalot (1986) \\
\hline $\begin{array}{l}\text { C. submolesta (Hustedt) Lange-Bertalot } \\
\text { (Figures 185-186) }\end{array}$ & L:17.0; W:3.8; S:23 & $4 a, 6 a b c$ & $6 \mathrm{c}$ & Krammer \& Lange-Bertalot (1986) \\
\hline Stauroneis anceps Ehrenberg (Figure 200) & $\begin{array}{l}\text { L:56.5-59.2; W:9.9-10.7; } \\
\text { S:20 }\end{array}$ & $5 \mathrm{c}$ & & Krammer \& Lange-Bertalot (1986) \\
\hline S. gracilis Ehrenberg (Figure 201) & L:88.7; W:15.9; S:19 & $1 \mathrm{~b}$ & & Reichardt (1995) \\
\hline \multicolumn{5}{|l|}{ Bacillariaceae } \\
\hline Nitzschia clausii Hantzsch (Figure 203) & $\begin{array}{l}\mathrm{L}: 28.2-41.2 ; \mathrm{W}: 4.9-5.3 ; \\
\mathrm{F}: 13\end{array}$ & $3 a$ & & Krammer \& Lange-Bertalot (1988) \\
\hline N. gracilis Hantzsch (Figures 196-197) & $\begin{array}{c}\mathrm{L}: 43.1-62.9 ; \mathrm{W}: 2.6-3.6 \\
\text { F:14 }\end{array}$ & $1 \mathrm{bc}$ & & Krammer \& Lange-Bertalot (1988) \\
\hline $\begin{array}{l}\text { N. intermedia Hantzsch ex Cleve \& Grunow } \\
\text { (Figures 198-199) }\end{array}$ & $\begin{array}{c}\mathrm{L}: 42.7-64.7 ; \mathrm{W}: 4-4.3 \\
\mathrm{~F}: 11-12\end{array}$ & $1 b, 2 b, 3 a, 5 c, 6 c$ & & Krammer \& Lange-Bertalot (1988) \\
\hline $\begin{array}{l}\text { N. palea (Kützing) W. Smith var. palea } \\
\text { (Figures 194-195) }\end{array}$ & $\begin{array}{c}\text { L: } 16.1-41.0 ; \mathrm{W}: 2.4-4.7 \\
\text { F: } 9-14\end{array}$ & $\begin{array}{l}1 \mathrm{bc}, 2 \mathrm{abc}, 3 \mathrm{ab}, 4 \mathrm{abc} \\
5 \mathrm{bc}, 6 \mathrm{abc}\end{array}$ & $1 b, 3 b, 5 b, 6 c$ & $\begin{array}{l}\text { Rumrich et al. (2000) } \\
\text { Levkov et al. (2007) }\end{array}$ \\
\hline $\begin{array}{l}\text { N. perminuta (Grunow) Peragallo } \\
\text { (Figures 190-191) }\end{array}$ & $\begin{array}{l}\mathrm{L}: 11.2-22.1 ; \mathrm{W}: 2.4-2.8 \\
\mathrm{~F}: 10-13\end{array}$ & $3 \mathrm{ab}, 4 \mathrm{~b}, 5 \mathrm{bc}, 6 \mathrm{~b}$ & & $\begin{array}{l}\text { Krammer \& Lange-Bertalot (1988), } \\
\text { Levkov et al. (2007) }\end{array}$ \\
\hline $\begin{array}{l}\text { N. vermicularis (Kützing) Hantzsch } \\
\text { (Figure 202) }\end{array}$ & L:106.3; W:4.9; F:10 & $4 \mathrm{~b}$ & & $\begin{array}{l}\text { Krammer \& Lange-Bertalot (1988), } \\
\text { Rumrich et al. (2000) }\end{array}$ \\
\hline \multicolumn{5}{|l|}{ Rhopalodiaceae } \\
\hline $\begin{array}{l}\text { Rhopalodia gibberula (Ehrenberg) O. Muller } \\
\text { (Figure 210) }\end{array}$ & L:36.1; W:8.6; S:19; A:9 & $6 \mathrm{~b}$ & & Krammer \& Lange-Bertalot (1988) \\
\hline \multicolumn{5}{|l|}{ Surirellaceae } \\
\hline $\begin{array}{l}\text { Stenopterobia curvula (W. Smith) Krammer } \\
\text { (Figure 206) }\end{array}$ & $\begin{array}{l}\text { L:127.7-156.4; } \\
\text { W:5.2; AC:7 }\end{array}$ & $1 \mathrm{~b}$ & & Metzeltin \& Lange-Bertalot (1998) \\
\hline $\begin{array}{l}\text { S. delicatissima (Lewis) Brébissoni } \\
\text { (Figure 207) }\end{array}$ & $\begin{array}{c}\text { L:60.4-75.6; } \\
\text { W:5.3-6.0; AC:7 }\end{array}$ & $4 b, 6 b$ & $6 \mathrm{c}$ & Metzeltin \& Lange-Bertalot (1998) \\
\hline Surirella angusta Kutzing (Figure 204) & $\mathrm{L}: 26.1 ; \mathrm{W}: 6.8 ; \mathrm{AC}: 7$ & 1a & & Krammer \& Lange-Bertalot (1988) \\
\hline $\begin{array}{l}\text { S. biseriata var. constricta Hustedt } \\
\text { (Figure 212) }\end{array}$ & L:190.4; W:17.6; AC:2 & $1 \mathrm{a}$ & & Huber-Pestalozii (1942) \\
\hline $\begin{array}{l}\text { S. lineares var. helvetica (Ehrenberg) Kützing } \\
\text { (Figure 208) }\end{array}$ & L:49.9; W:14.5; AC:3 & $4 \mathrm{~b}$ & & Metzeltin \& Lange-Bertalot (1998) \\
\hline S. splendida (Ehrenberg) Kutzing (Figure 211) & L: 113; W: 40.3: AC:2 & 1a & & Krammer \& Lange-Bertalot (1988) \\
\hline S. tenuissima Hustedt (Figure 205) & $\mathrm{L}: 25.3 ; \mathrm{W}: 8.5 ; \mathrm{AC}: 4$ & 1a & & $\begin{array}{l}\text { Simonsen (1987), Krammer \& } \\
\text { Lange-Bertalot (1988) }\end{array}$ \\
\hline Surirella sp. (Figure 209) & $\mathrm{L}: 57.2 ; \mathrm{W}: 9.5 ; \mathrm{AC}: 4$ & $6 \mathrm{c}$ & & \\
\hline
\end{tabular}

\section{Results and Discussion}

A total of 135 infrageneric diatoms taxa were identified (Table 3, Figures 2-222), representing eighteen families (Round et al. 1990, Kulikovskiy et al., 2014). The species richness from Piraquara II reservoir was higher than the diatom floras found in the nearby urban reservoirs Iraí (96 taxa), Passaúna (106 taxa) and Itaqui (124 taxa) (Silva et al. 2010, Bertolli et al. 2010, Faria 2010). In June, (127 taxa) it was found higher richness than in November (66 taxa).

The diatoms occuring highly frequent were (10 taxa): Achnanthidium caledonicum (Lange-Bertalot) Lange-Bertalot, Achnanthidium macrocephalum
(Hustedt) Round \& Bukhtiyarova, Achnanthidium minutissimum (Kützing) Czarnecki, Aulacoseira tenella (Nygaard) Simonsen, Brackysira neoexilis Lange-Bertalot, Discostella stelligera (Cleve \& Grunow) Houk \& Klee, Eunotia pseudosudetica Metzeltin, Lange-Bertalot \& García-Rodríguez, Eunotia intermedia (Krasske) Nörpel-Schempp \& Lange-Bertalot, Fragilaria recapitellata Lange-Bertalot \& Metzeltin and Fragilaria parva (Grunow) Tuji $\&$ Williams. Diatoms registered as frequent were (11 taxa): Achnanthidium catenatum (Bily \& Marvan) Lange-Bertalot, Achnanthidium eutrophilum (Lange-Bertalot) Lange-Bertalot, Aulacoseira ambigua (Grunow) Simonsen f. ambigua, Aulacoseira granulata (Ehrenberg) Simonsen var. granulata, Eunotia bilunaris (Ehrenberg) Mills, Encyonema neogracile Krammer, 

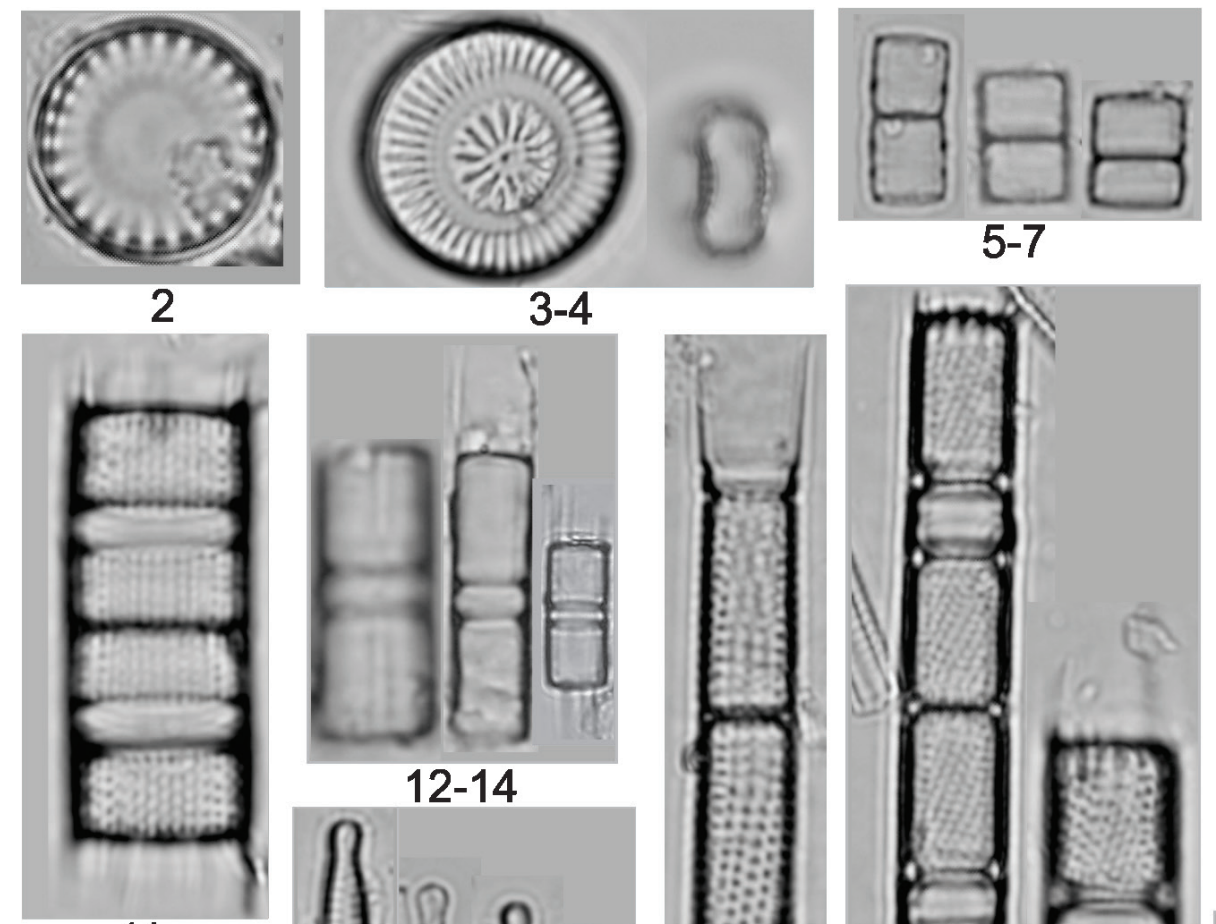

11
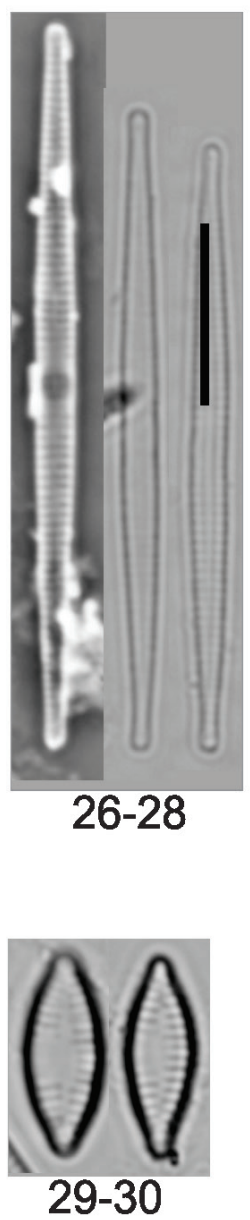
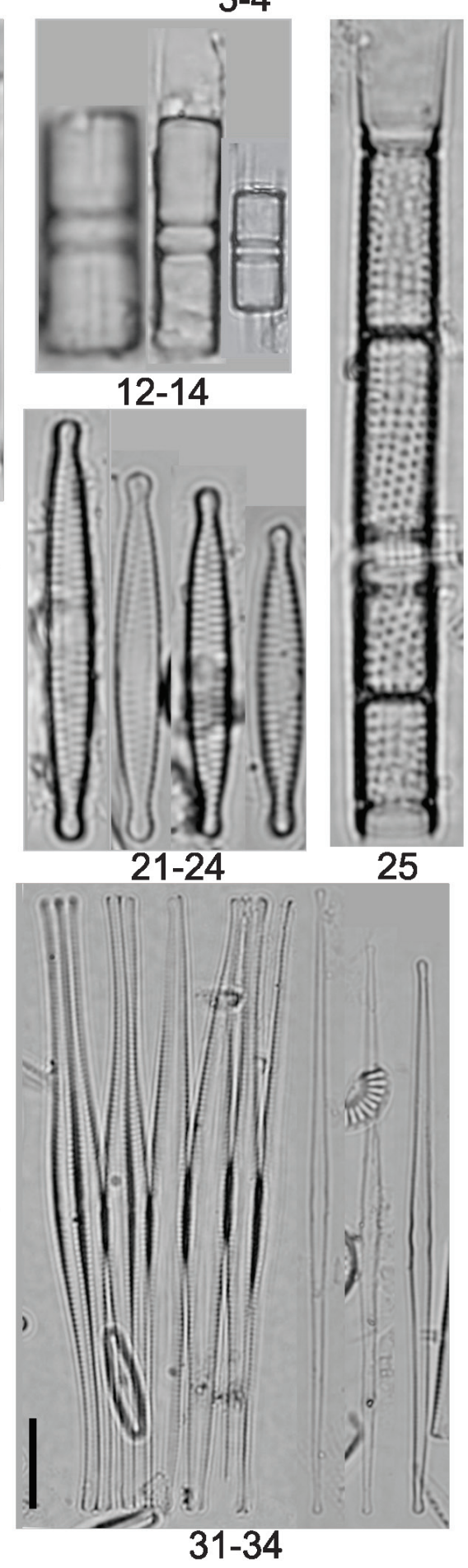
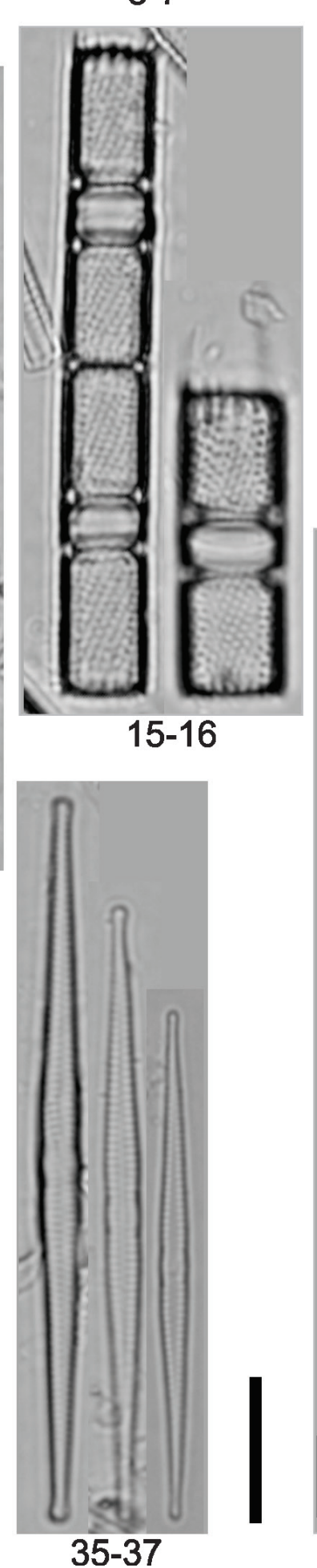
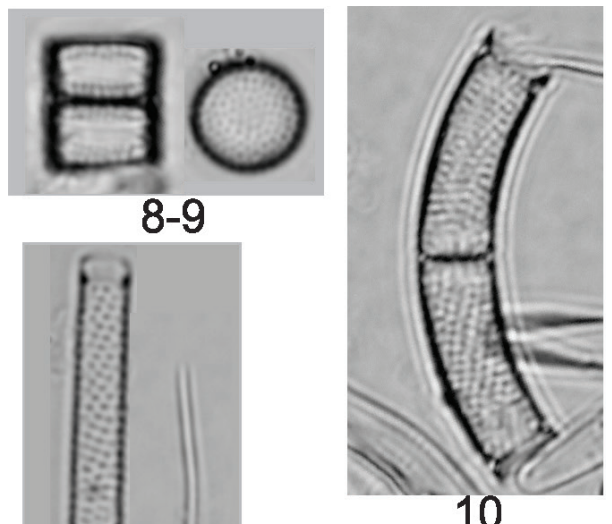

10
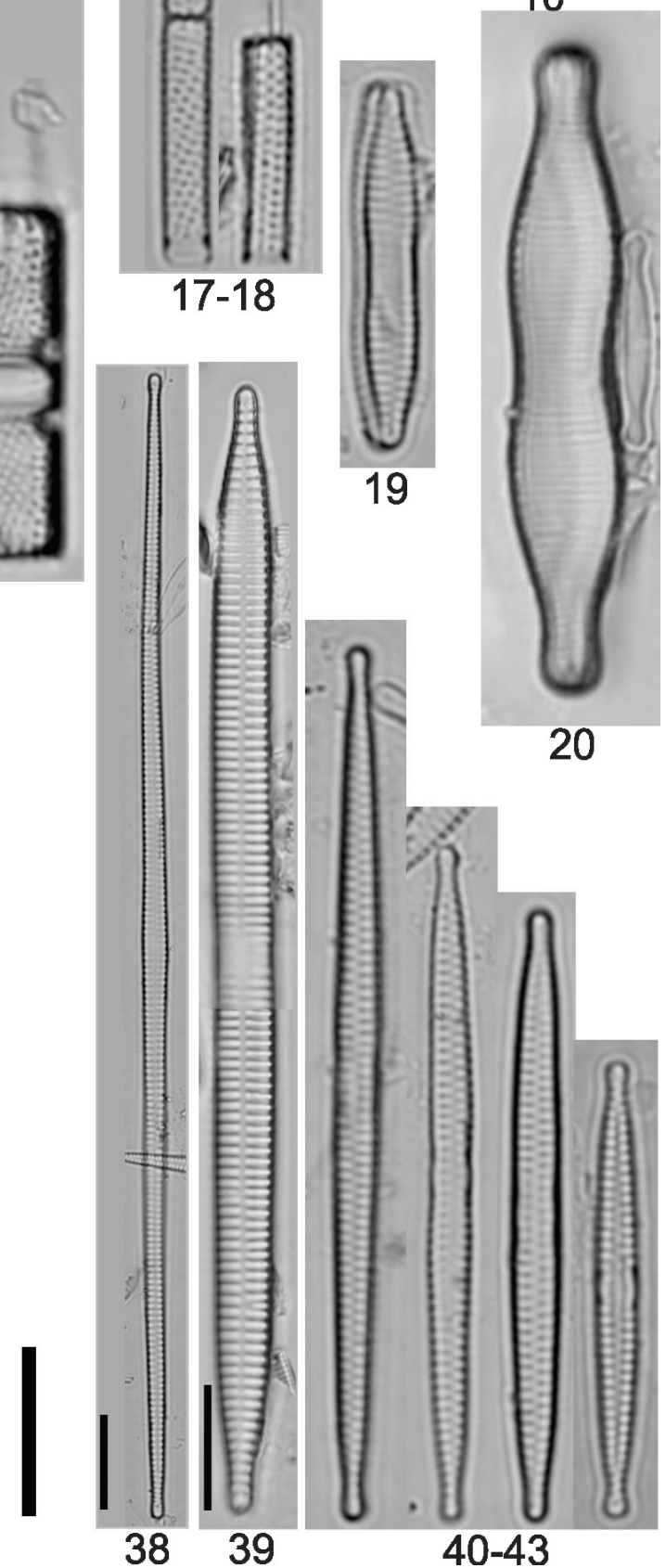

Figures 2-43. Diatoms from Piraquara II reservoir. 2. Cyclotella meneguiniana. 3-4. Discostella stelligera. 5-7. Aulacoseira sp. 8-9. A. tenella. 10. A. ambigua var. ambigua f. spiralis. 11. A. brasiliensis. 12-14. A. herzogii var. herzogii. 15-16. A. ambigua var. ambigua f. ambigua. 17-18. A. granulata var. angustissima. 19. Fragilaria mesolepta. 20. Fragilariforma javanica. 21-24. Fragilaria pectinalis. 25. Aulacoseira granulata var. granulata. 26-28. Fragilaria gracilis. 29-30. F. microvaucheriae. 31-34. F. crotonensis. 35-37. F. tenera. 38. Ulnaria acus. 39. U. ulna. 40-43. Fragilaria parva. Scales: $10 \mu \mathrm{m}$. 

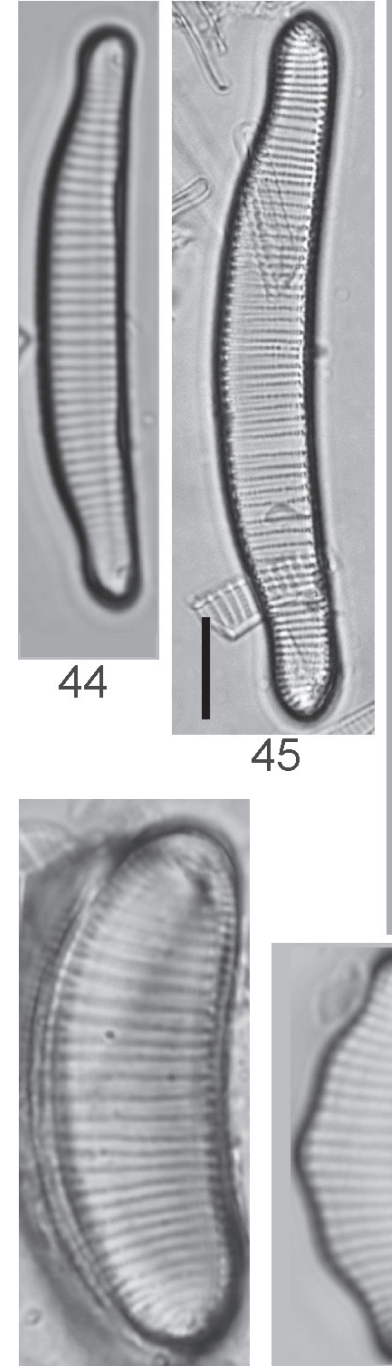

59
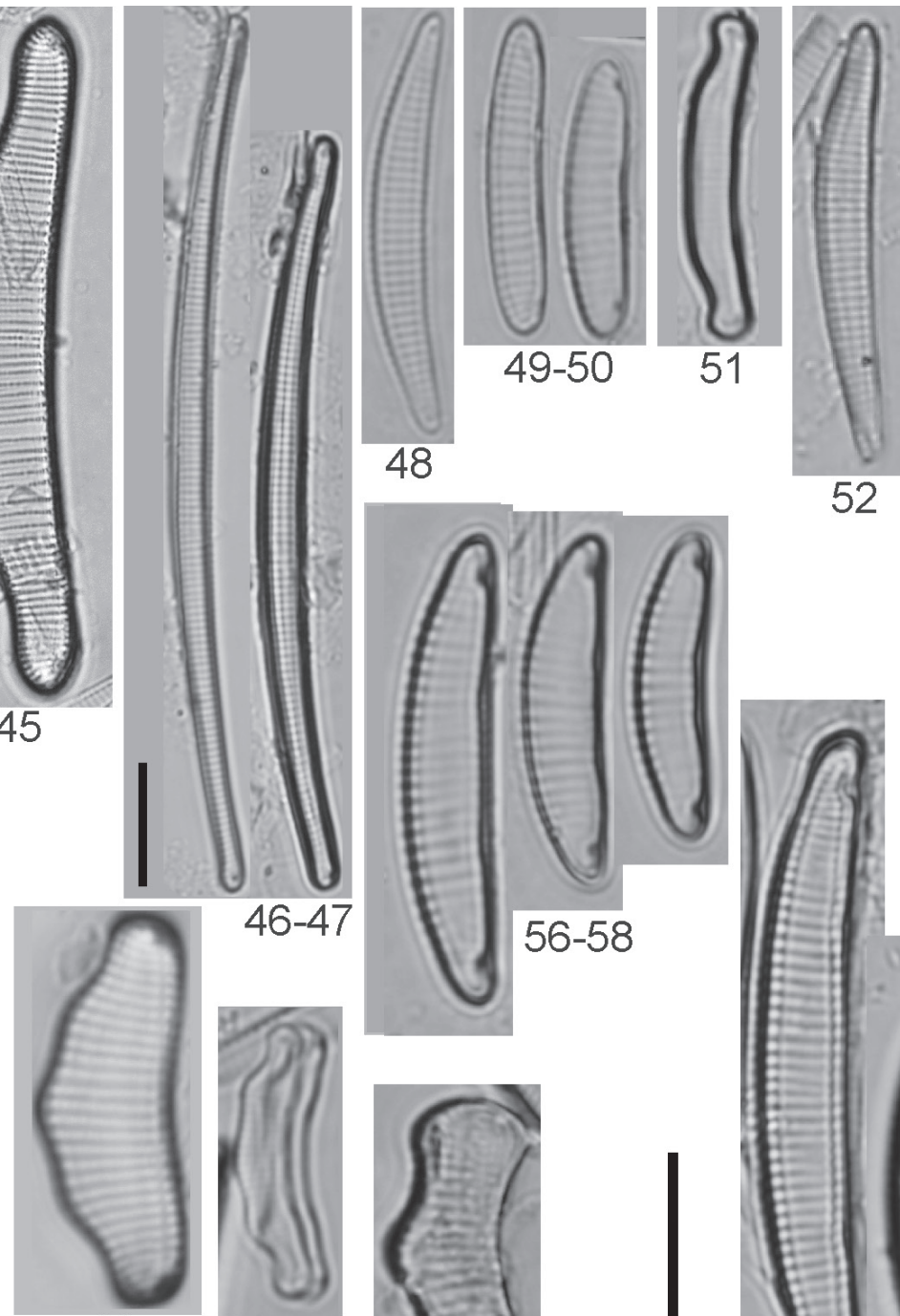

60

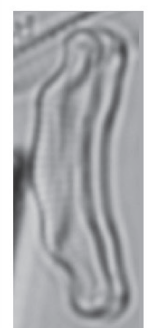

61
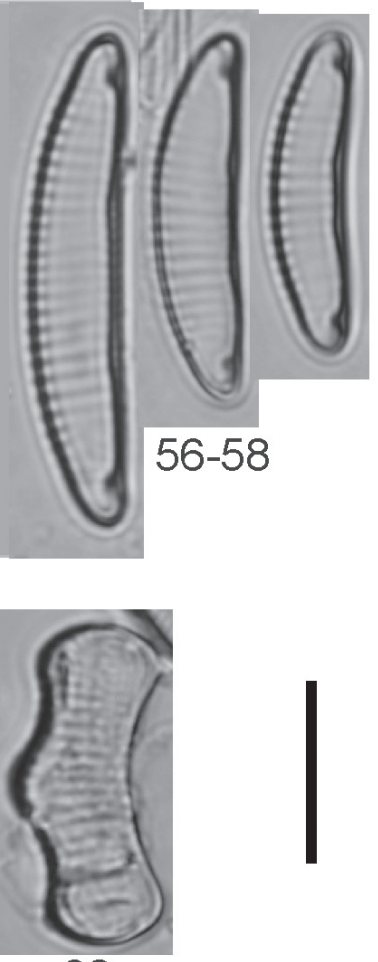

62

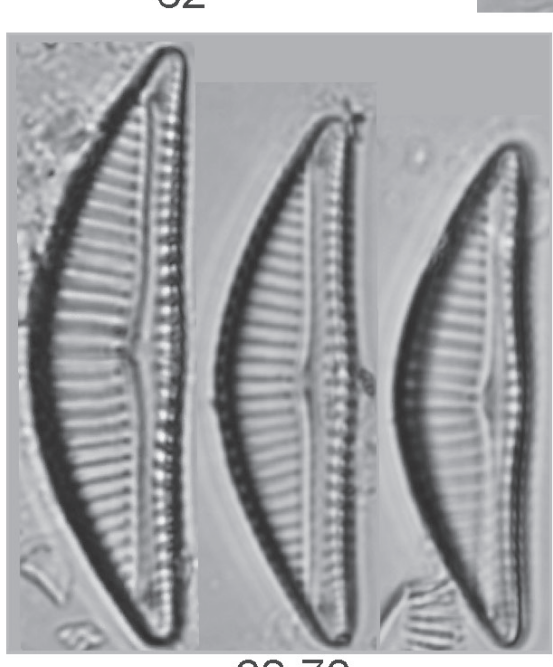

68-70
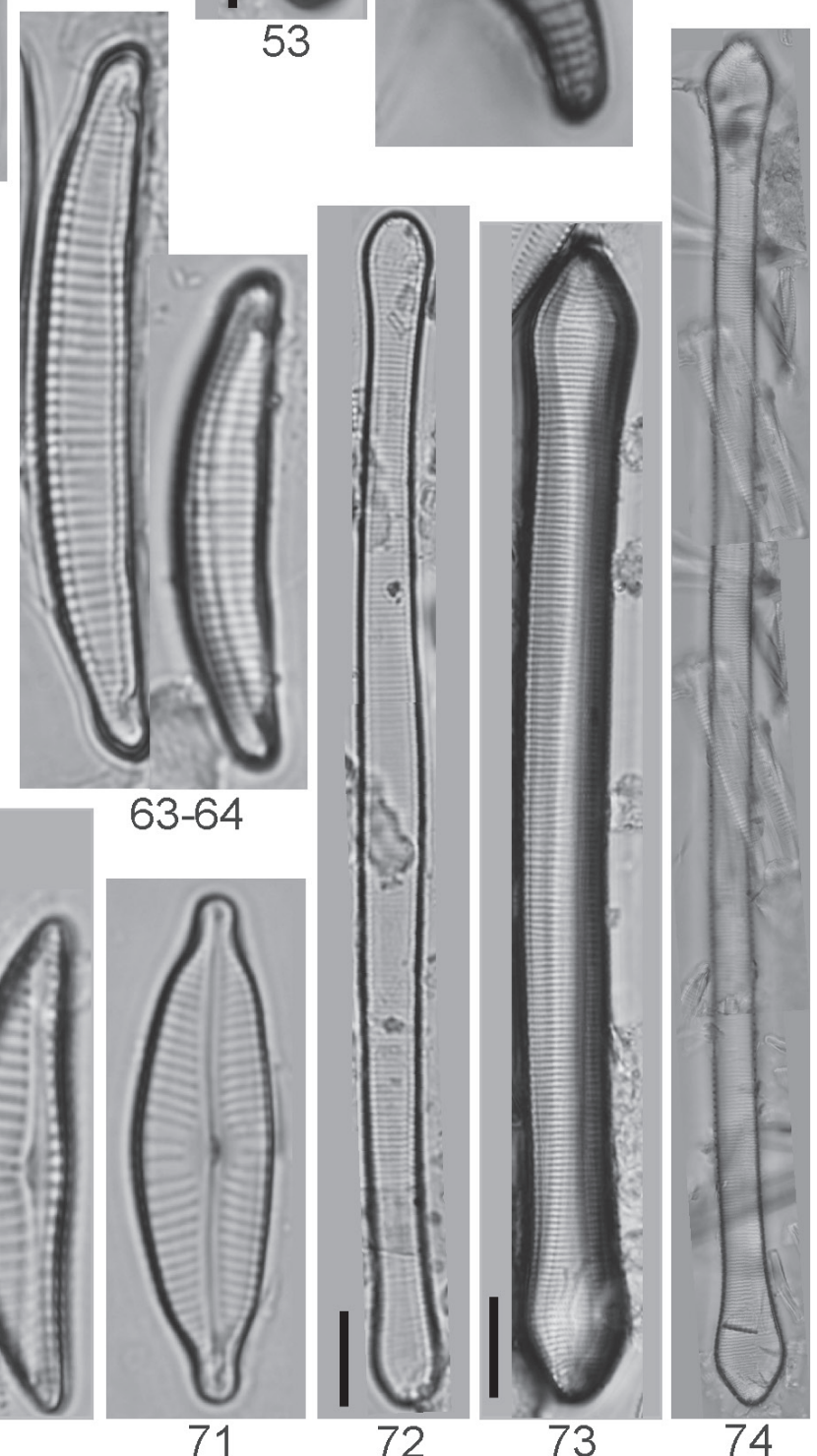

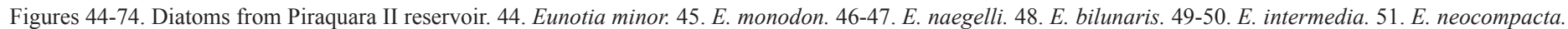

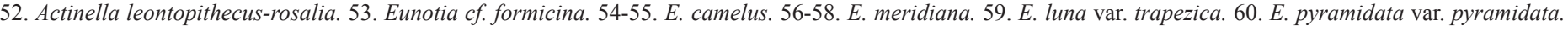

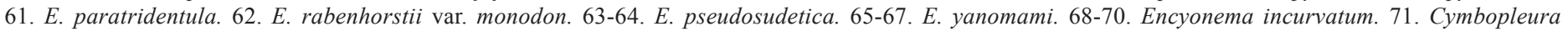
naviculiformis.72. Eunotia desmogonioides. 73. D. ossiculum. 74. D. transfugum. Scales: $10 \mu \mathrm{m}$. 

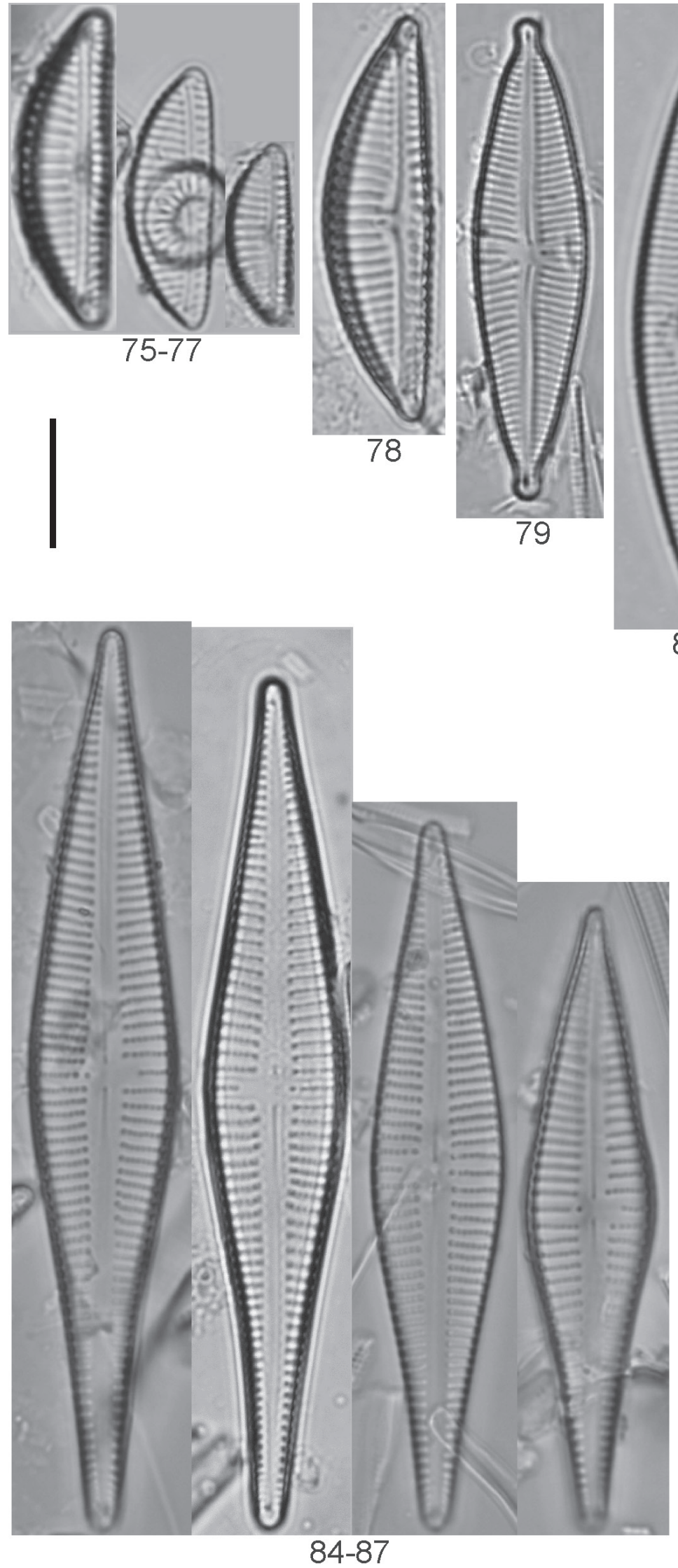
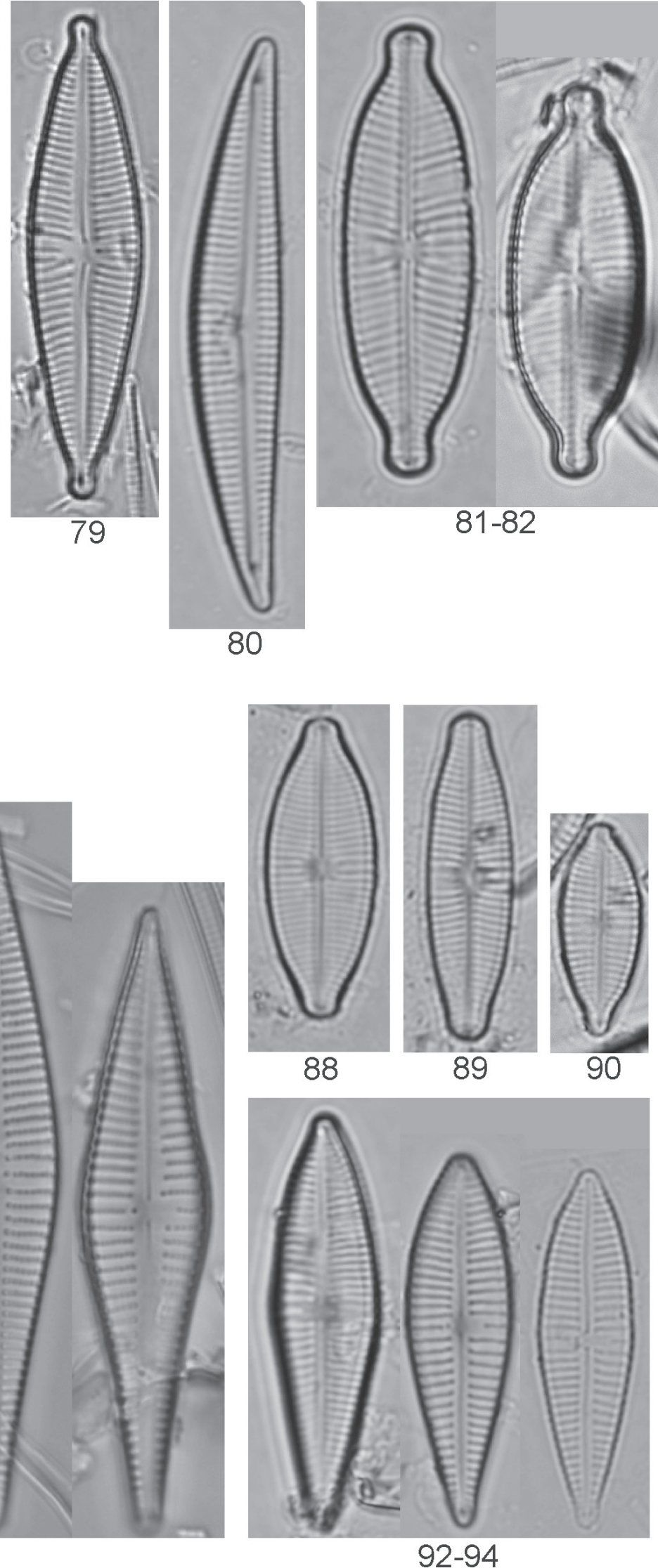

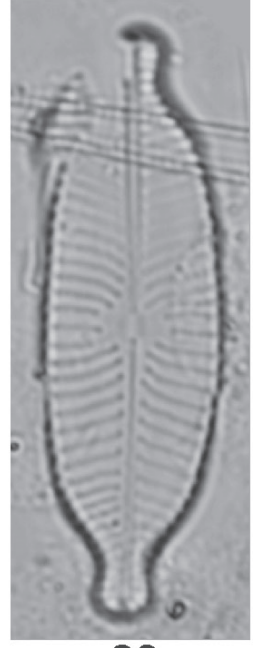

83

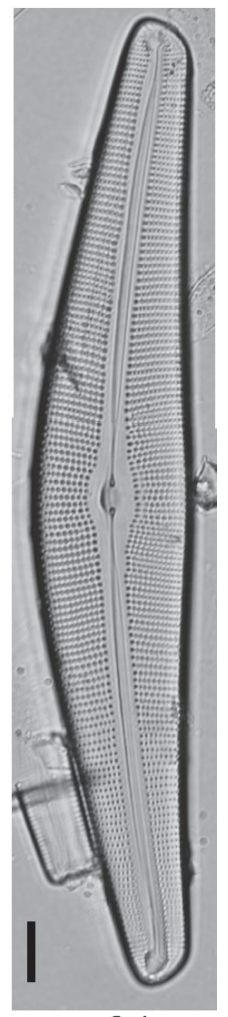

91

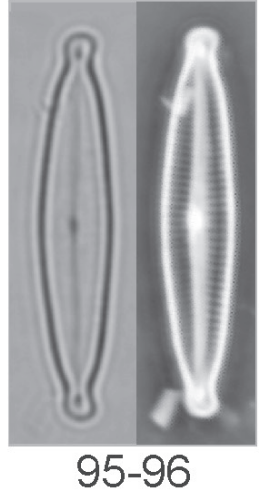

Figures 75-96. Diatoms from Piraquara II reservoir. 75-77. Encyonema silesiacum. 78. E. vulgare var. vulgare. 79. Encyonopsis frequentiformis. 80. Encyonema neogracile. 81-82. Placoneis elginensis. 83. P. symmetrica. 84-87. Gomphonema guaraniarum. 88. Geissleria punctifera. 89. G.lateropunctata. 90. Gomphonema parvulum var. subcapitata. 91. Cymbella aspera. 92-94. Gomphonema pseudoargur. 95-96. Encyonopsis microcephala. Scales: $10 \mu \mathrm{m}$. 

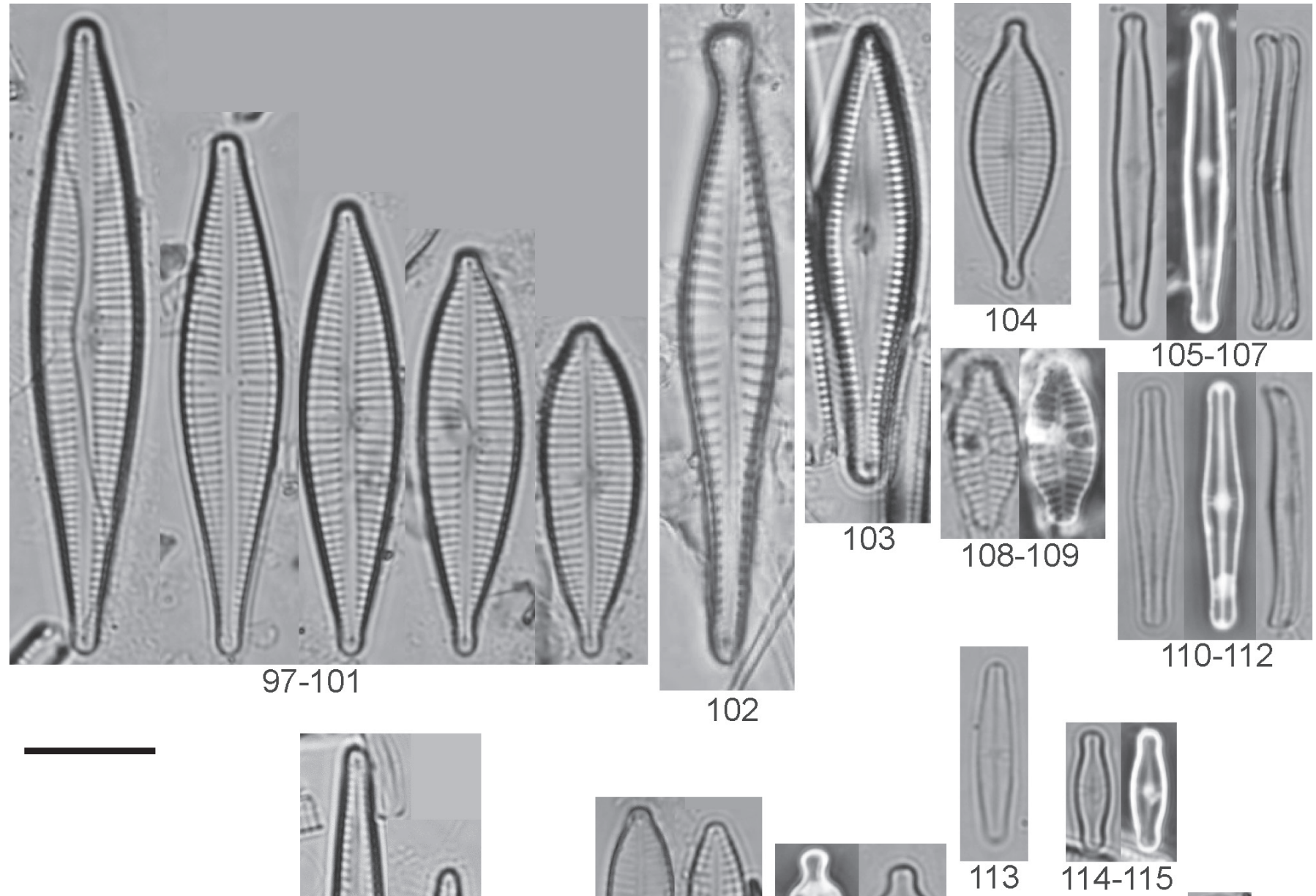

$110-112$

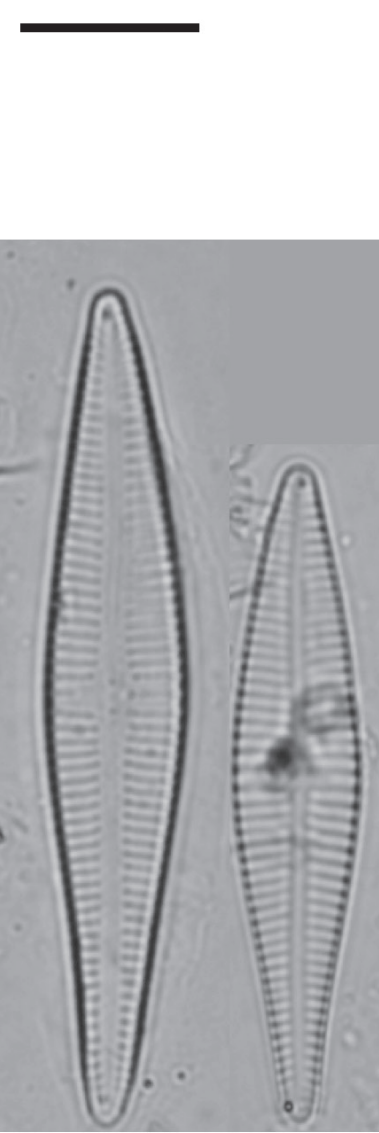

116-117
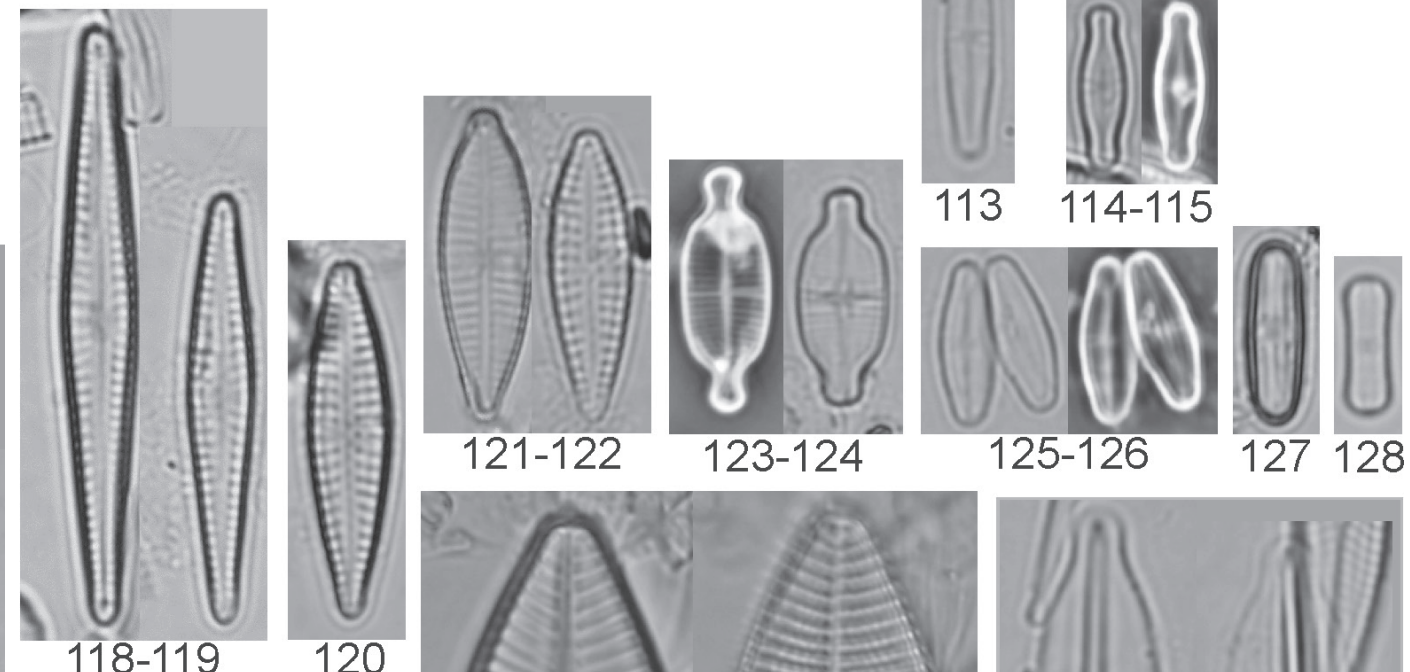

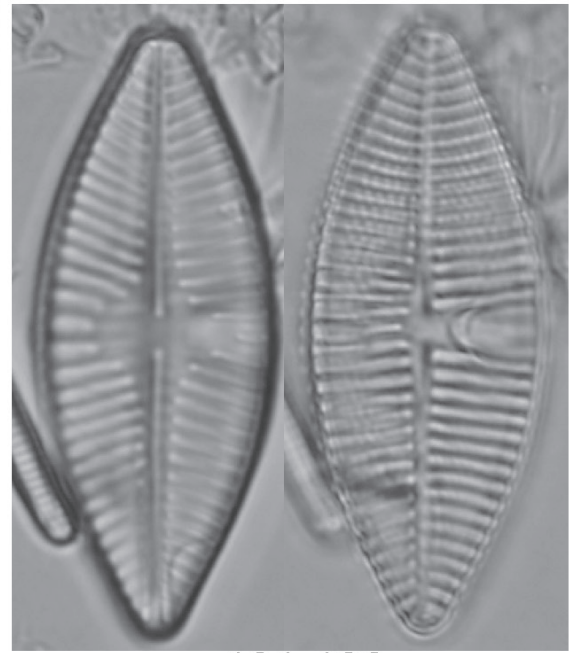

$131-132$

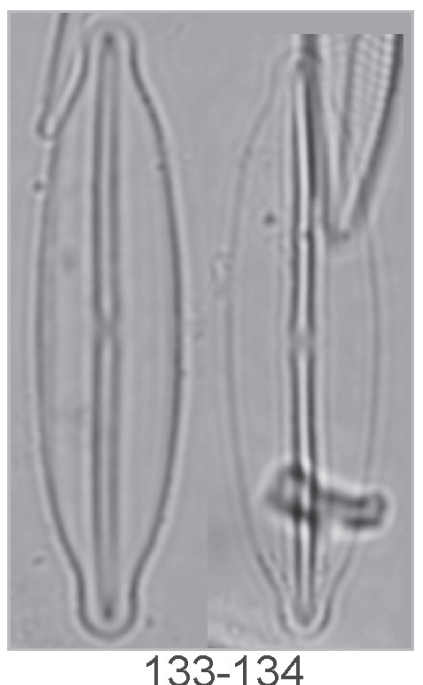

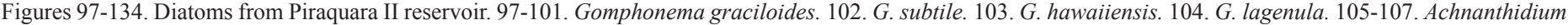

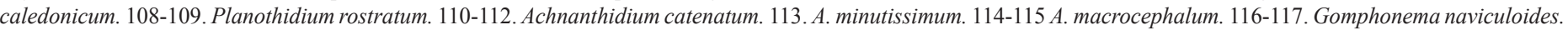

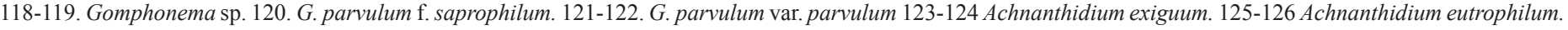

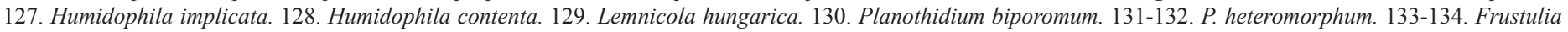
crassinervia. Scales: $10 \mu \mathrm{m}$. 

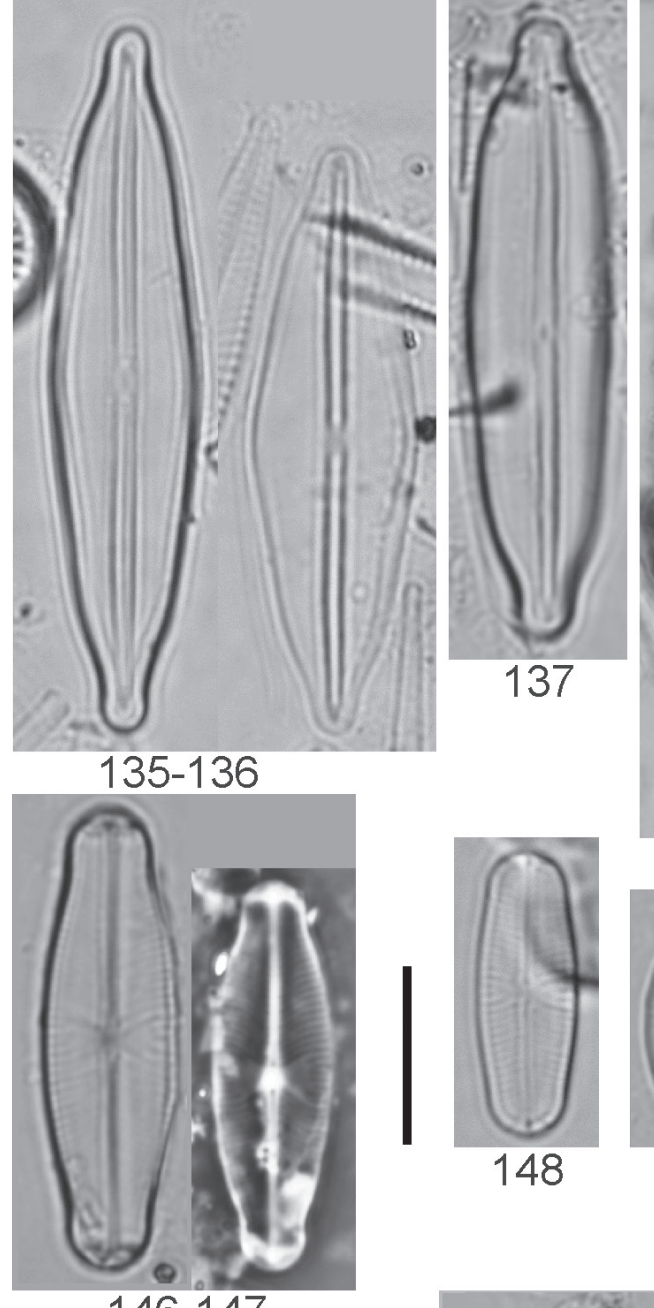

137
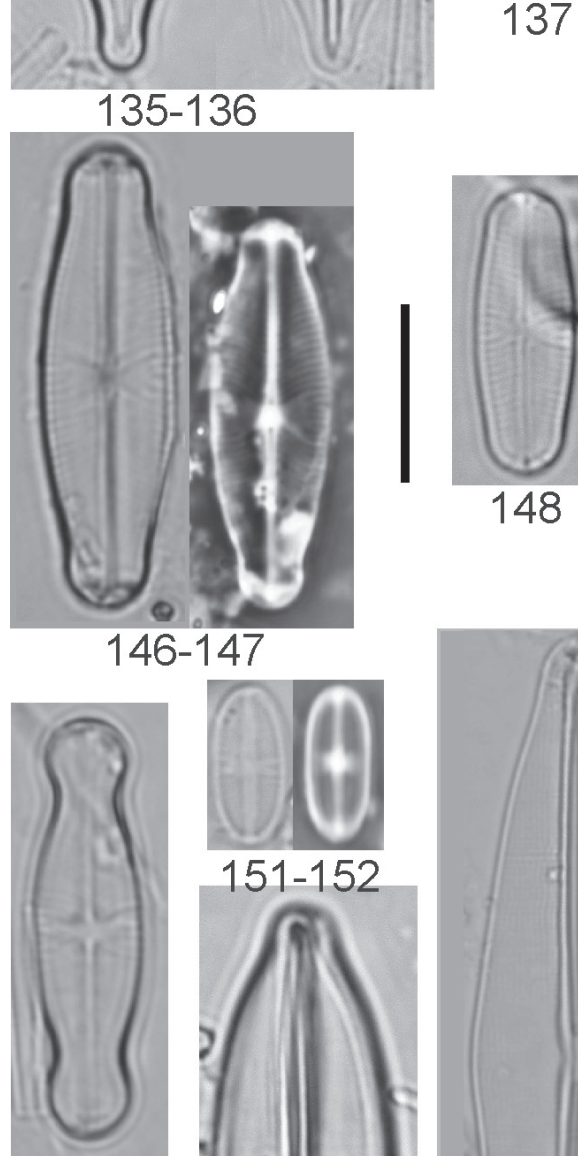

153

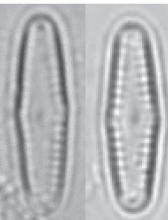

154-155

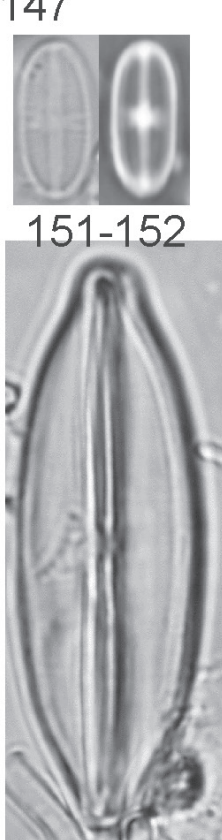

156

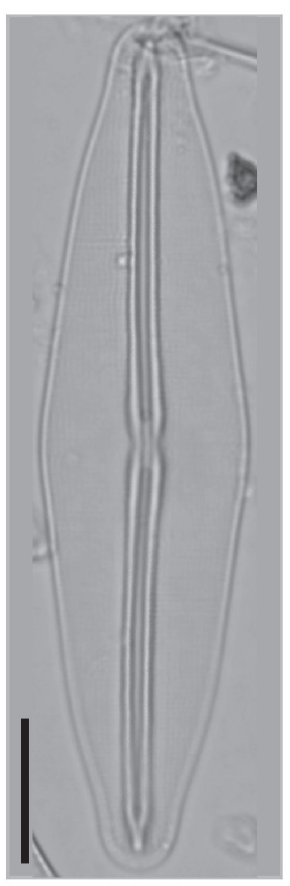

157

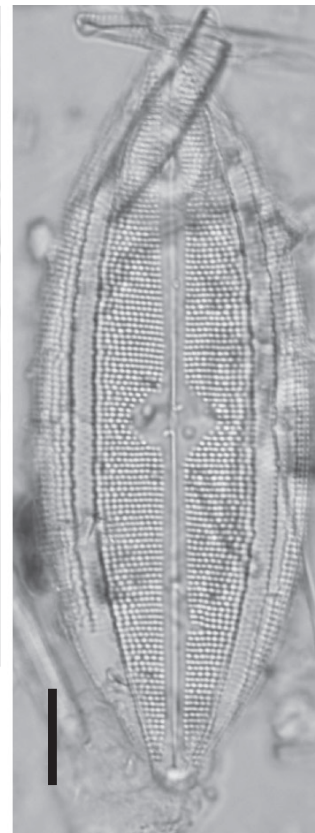

138

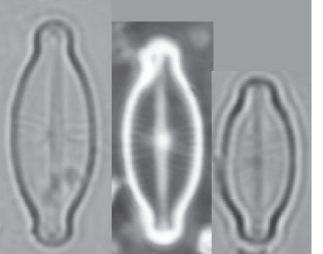

$149-150$

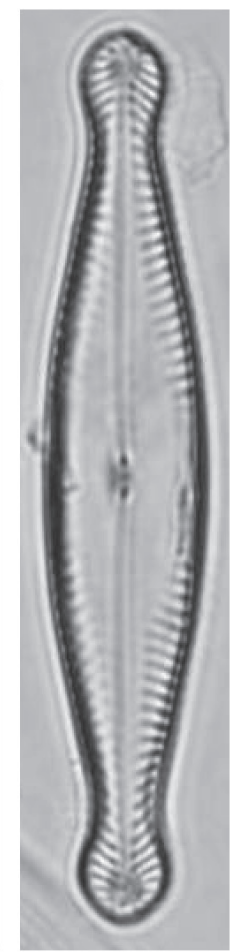

158
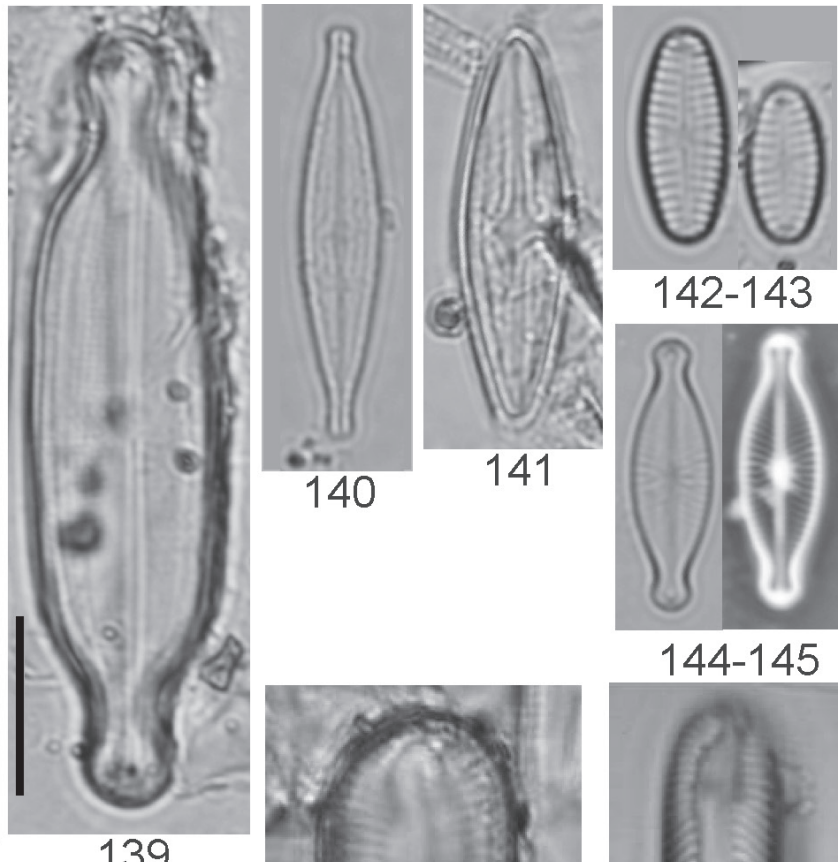

144-145

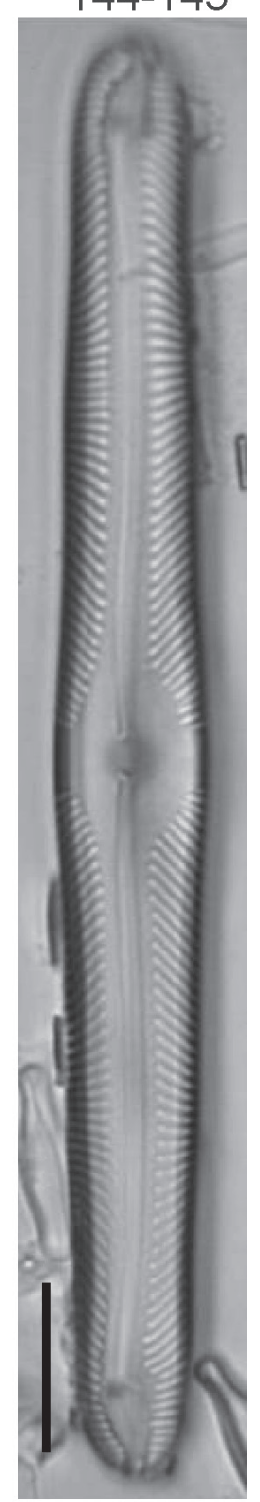

161

Figures 135-161. Diatoms from Piraquara II reservoir. 135-136. Frustulia undosa. 137. F. guayanensis. 138. Neidium iridis. 139. N. affine. 140. B. brebissoni. 141. Brackysira neoexilis. 142-143 Sellaphora saugerresii. 144-145. S. sassiana. 146-147. S. pupula. 148. Sellaphora sp. 149-150. S. sardiniensis. 151-152. S. nigri. 153. S. ventraloconfusa. 154-155. Chamaepinnularia mediocris. 156. Frustulia acidophilissima. 157. F. quadrisinuata. 158. Pinnularia acrosphaeria. 159. P. latarea. 160. P. brauniana. 161. P. stoermeri. Scales: $10 \mu \mathrm{m}$. 


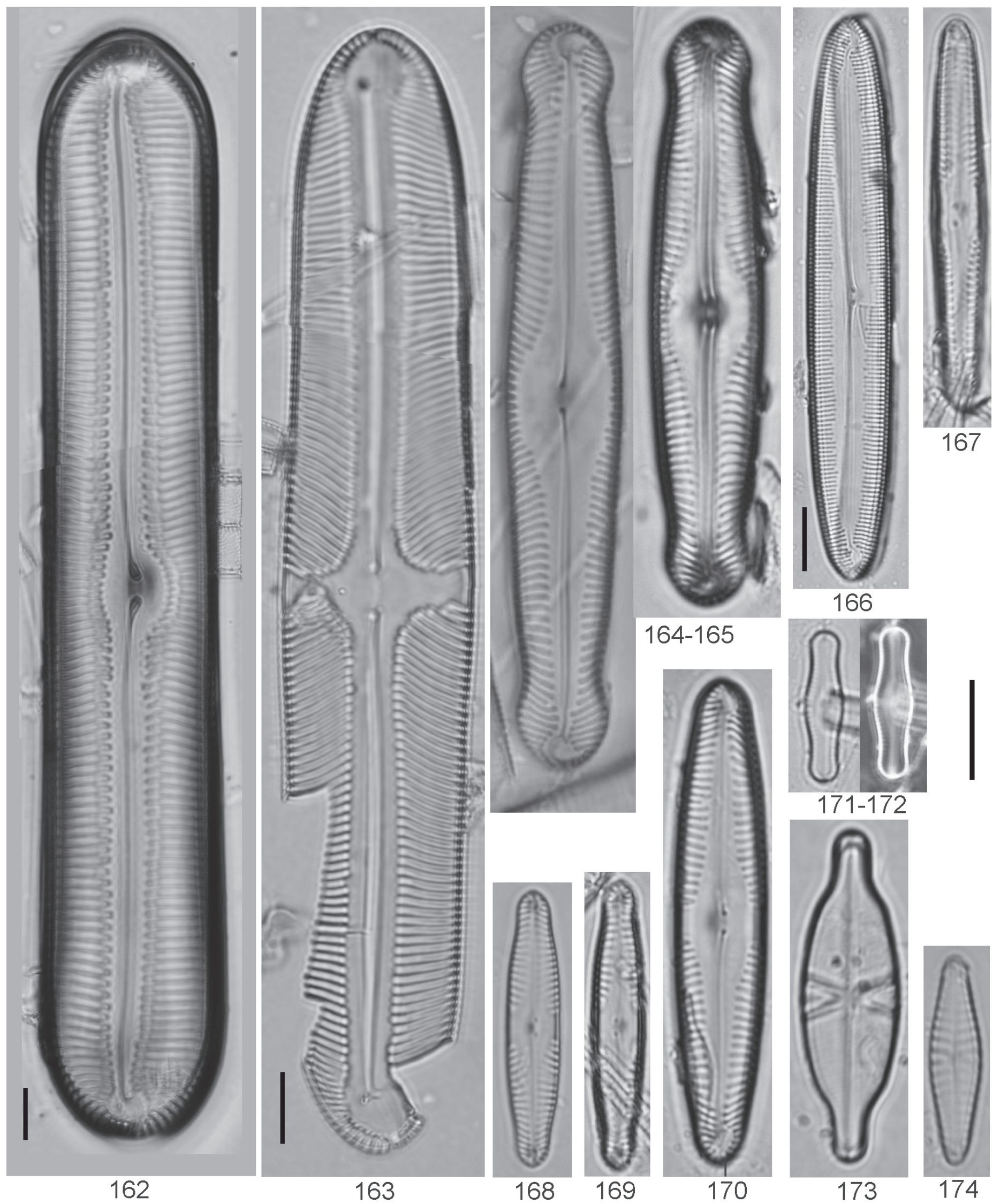

Figures 162-174. Diatoms from Piraquara II reservoir. 162. Pinnularia latevitatta. 163. P. subgibba var. undulata. 164-165. P. gibba. 166. P. butantanum. 167. P. similiformis. 168. P. divergentissima var. minor. 169. P. subcapitata. 170. P. subbrevistriatta. 171-172 Chamaepinnularia brasilianopsis. 173. Capartogramma crucicola. 174. Hippodonta capitatta ssp. iberoamericana. Scales: $10 \mu \mathrm{m}$. 

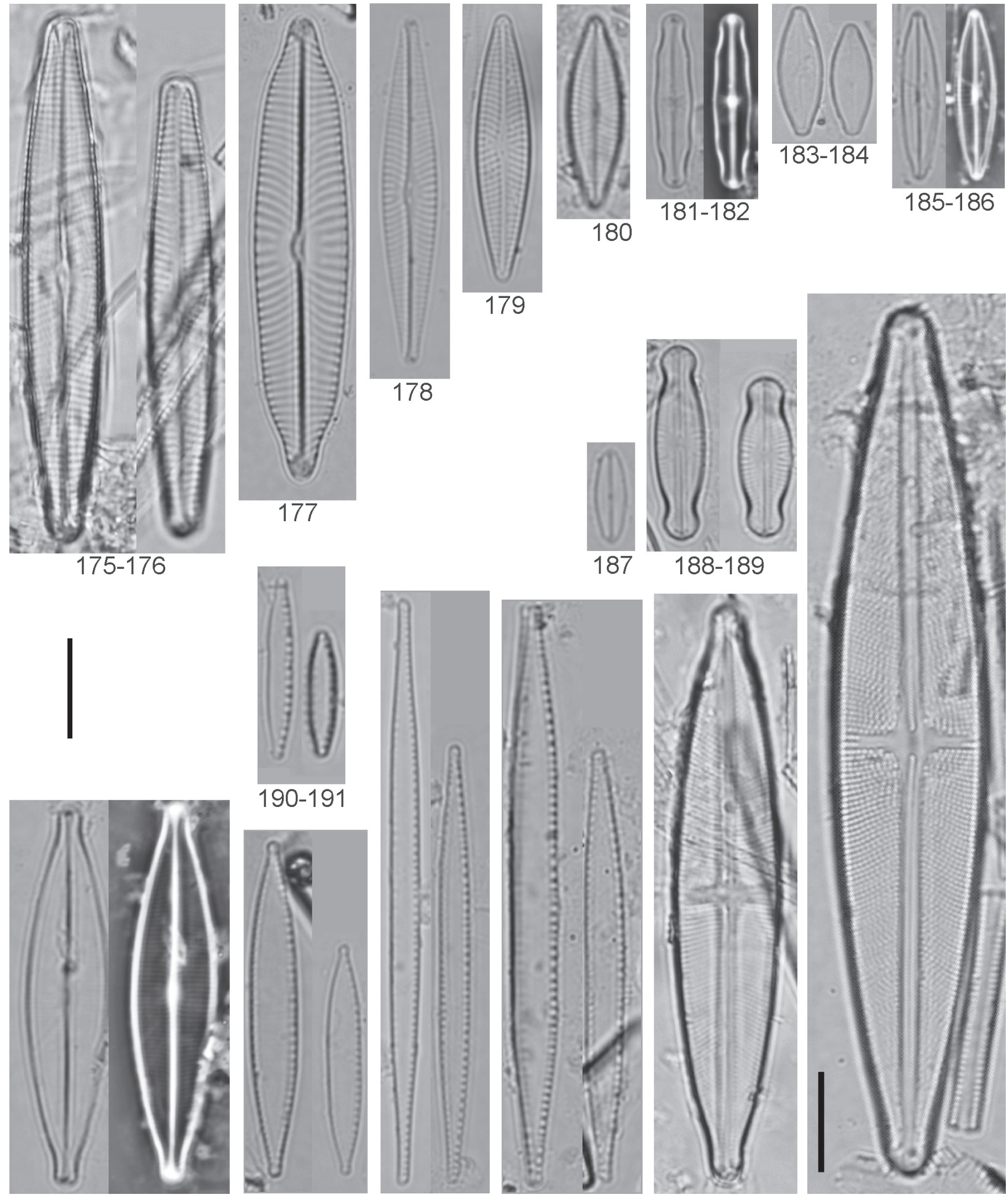

192-193

$190-191$

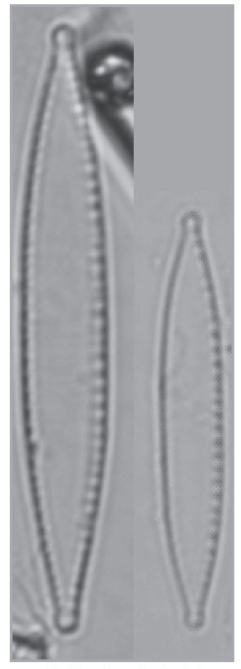

194-195
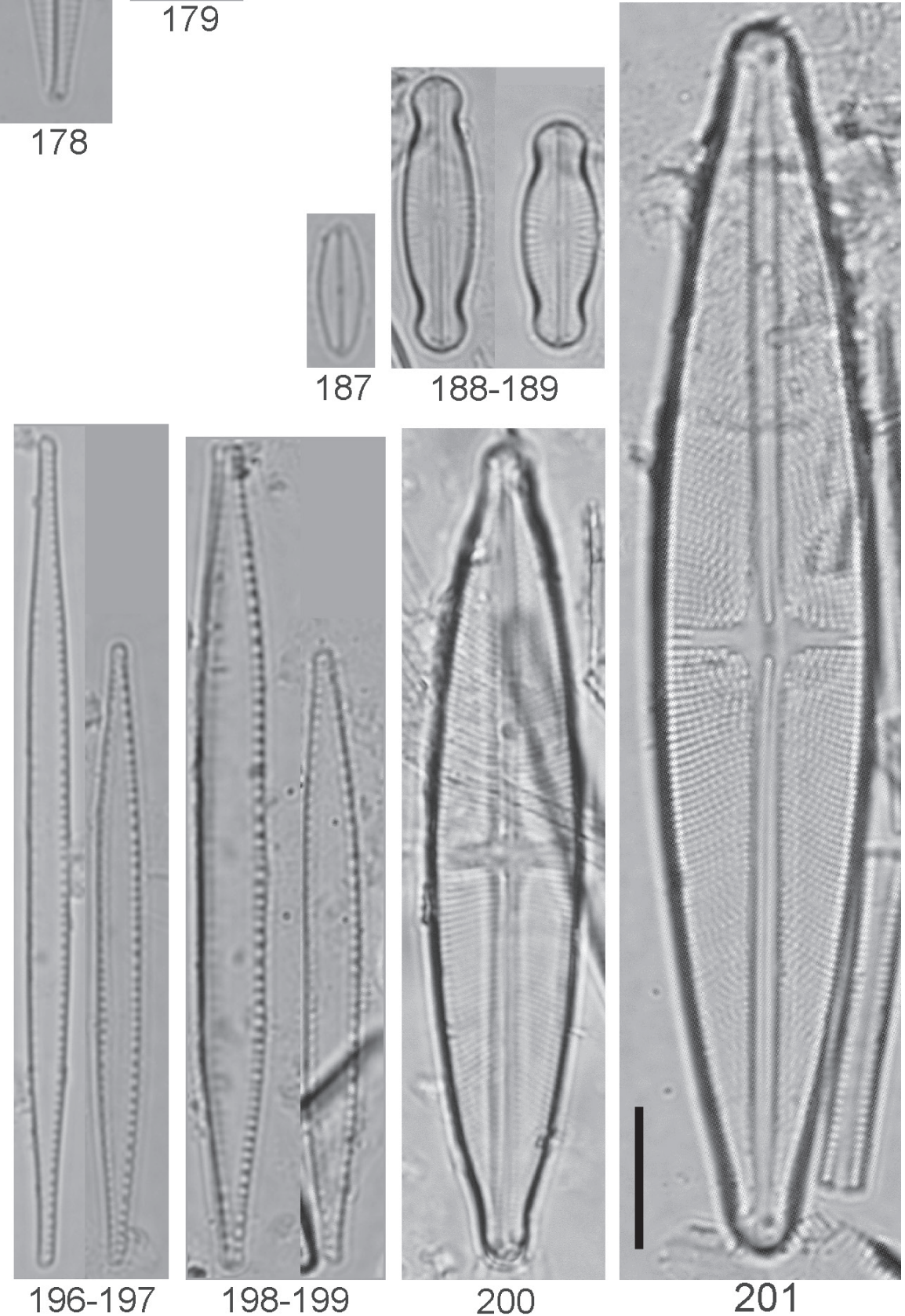

Figures 175-201. Diatoms from Piraquara II reservoir. 175-176. Navicula angusta. 177. N. viridulacalcis. 178. N. notha. 179. N. cryptotenella. 180. N. veneta. 181-182. N. tridentula. 183-184. Nupela torganiae. 185-186. Craticula submolesta. 187. Mayamaea permitis. 188-189. Naviculadicta ventraloconfusa var. chilensis. 190-191. Nitzschia perminuta. 192-193. Craticula riparia. 194-195. Nitzschia palea. 196-197. N. gracilis. 198-199. N. intermedia. 200. Stauroneis anceps. 201. S. gracilis. Scales: $10 \mu \mathrm{m}$. 

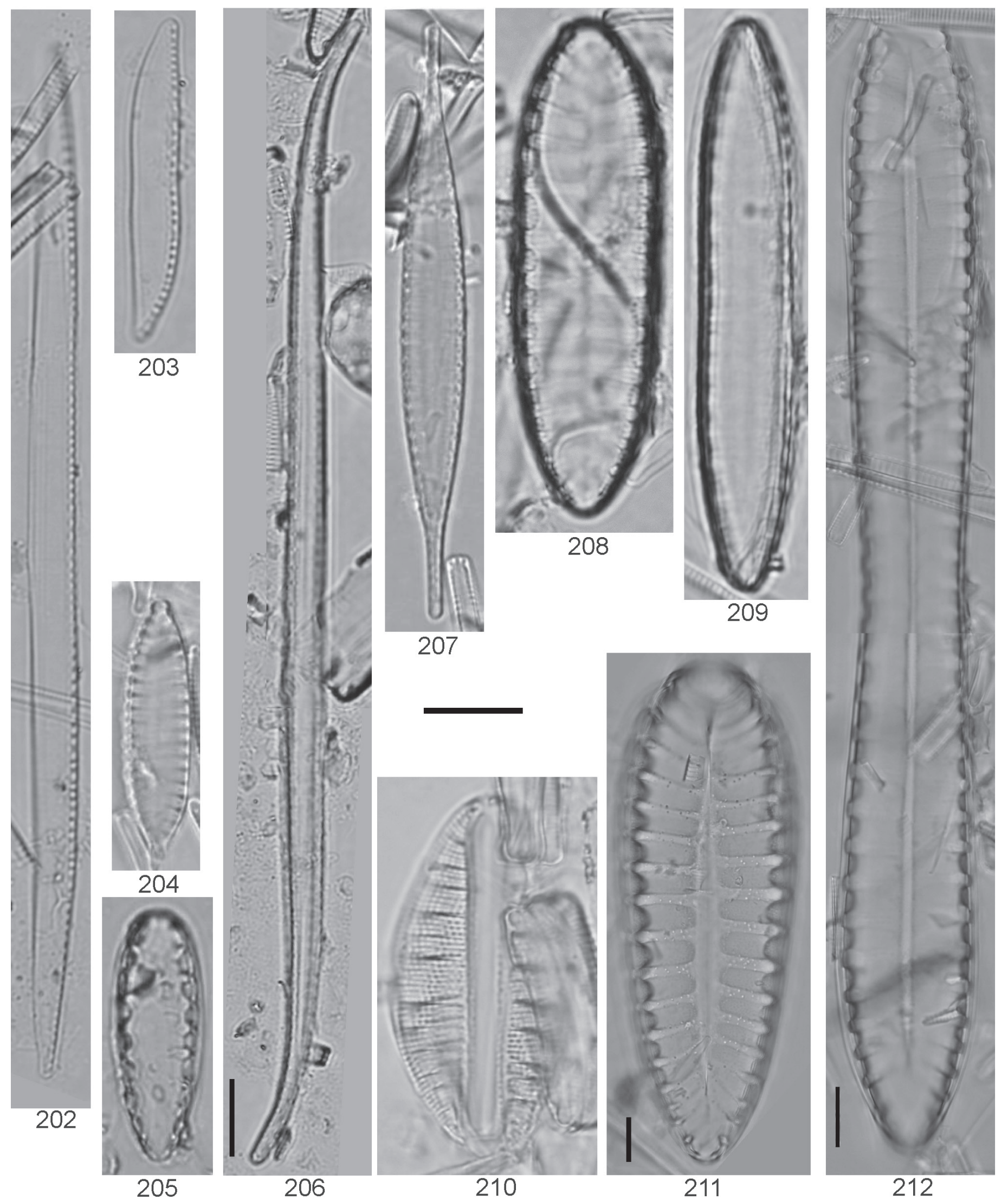

212

Figures 202-212. Diatoms from Piraquara II reservoir. 202. Nitzschia vermicularis. 203. N. clausii. 204. Surirella angusta. 205. S. tenuissima. 206. Stenopterobia curvula. 207. S. delicatissima. 208. Surirella lineares var. helvetica. 209. Surirella sp. 210. Rophalodia gibberula. 211. Surirella splendida. 212. S. biseriata var. constricta. Scales: $10 \mu \mathrm{m}$. 


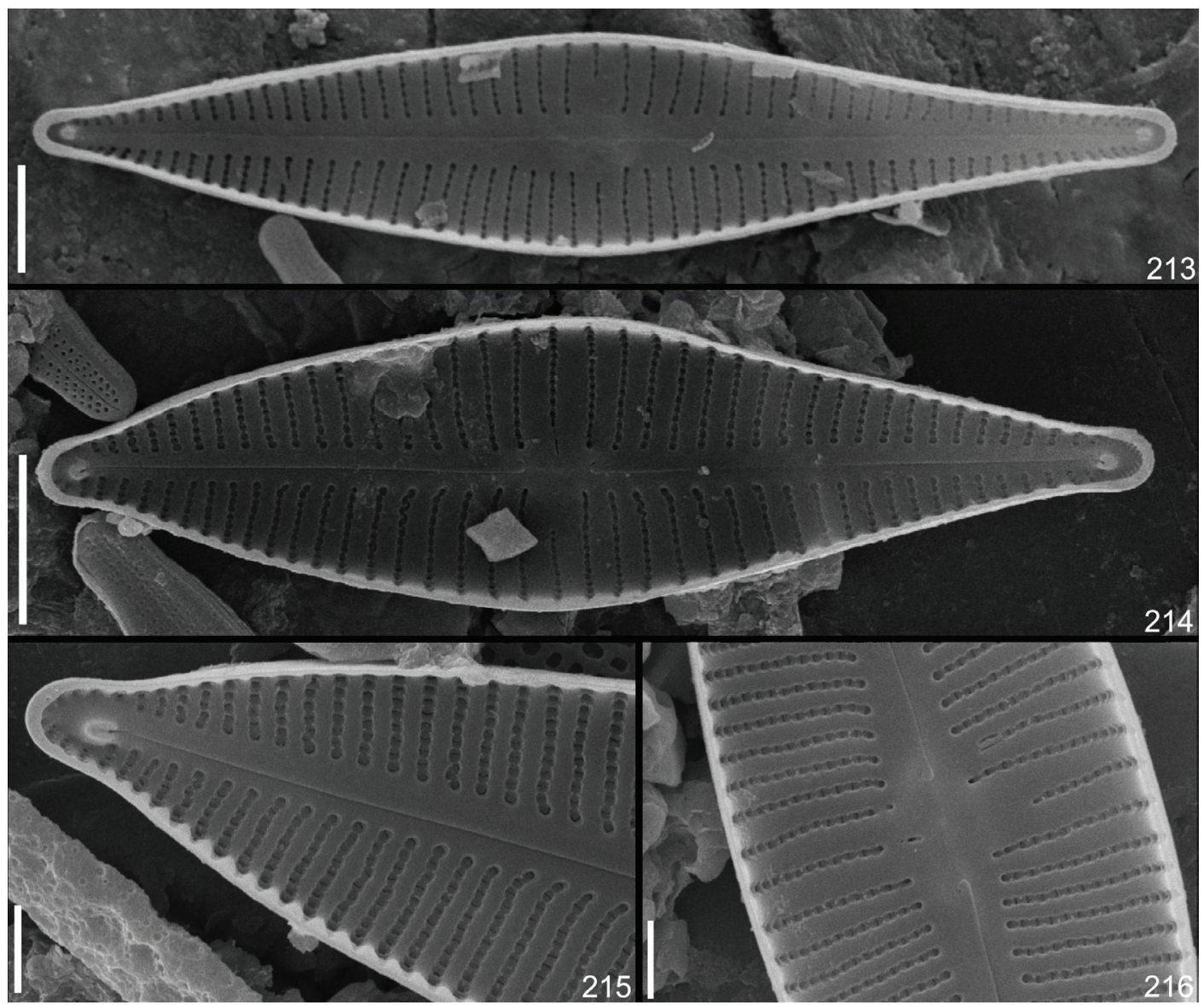

Figures 213-216. Diatoms from Piraquara II reservoir (SEM). 213. Internal view of Gomphonema naviculoides, scale: $5 \mu \mathrm{m}$. 214. Internal view of G. graciloides, scale: $5 \mu \mathrm{m}$. 215. Headpole of G. graciloides in internal view, scale: $1 \mu \mathrm{m}$. 216. Median region of G. graciloides showing the proximal raphe ends and the stigma opening in internal view, scale: $2 \mu \mathrm{m}$.

Fragilaria tenera (W. Smith) Lange-Bertalot, Fragilaria microvaucheriae Wetzel \& Ector, Gomphonema graciloides Hustedt, Gomphonema lagenula Kützing and Nitzschia palea (Kützing) W. Smith. Altogether the more frequent diatoms totalized $15.3 \%$ of 135 taxa identified and $54.7 \%$ were sporadic in the reservoir.

Achnanthidium minutissimum and Brachysira neoexilis were the most frequent taxa present in $90 \%$ of the samples. Among the very frequent diatoms we found other species included in Achnantidium, Fragilaria and Eunotia. The solitary Discotella stelligera and the short chain Aulacoseira tenella are free living species that entangled among diatoms from the biofilm.

Description and comments of specimens not identified and first registered to the state of Paraná are bellow.

\section{Aulacoseiraceae}

\section{Aulacoseira sp.}

Figs 5, 7

Frustules solitary or in short chains. Valves circular, shallow ringleist; inconspicuous striae, areolae and spines.

Aulacoseira sp. resembles A. simoniae Tremarin, Torgan \& Ludwig and $A$. tenella, differing by the conspicuous ornamentation of the latter species (Tremarin et al. 2014). Specimens were rare in samples, making detailed analysis impracticable.

\section{Eunotiaceae}

Eunotia cf. formicina Lange-Bertalot in Lange-Bertalot et al., Diatoms of Europe 6: 105; pl. 222, figs 1-7, pl. 223, 2011.
Fig. 53

Valves with dorsal margin convex, ventral margin concave, ends rounded, not detached from the valve, striae parallel to radiate near the apices.

Eunotia formicina was recently proposed (Lange-Bertalot et al. 2011) to nominate morphotypes of $E$. formica Ehrenberg distinguished by rounded ends, narrower valve $(6-8 \mu \mathrm{m})$ and denser areolae $(25-28$ in $10 \mu \mathrm{m})$ in the striae ( 8 -12 in $10 \mu \mathrm{m})$. Eunotia formica shows cuneate poles, wider valves (7-14 $\mu \mathrm{m})$ and less denser striae $(6-12$ in $10 \mu \mathrm{m})$. Central delicate gibbosity at ventral margin of E. formicina was not observed in our specimens and the striae are originally less dense.

\section{Gomphonemataceae}

Gomphonema guaraniarum Metzeltin \& Lange-Bertalot in Lange-Bertalot, Iconogr. Diatomol. 18: 147, pl. 212, figs 9-14, 2007.

Figs 84-87

Valves rhombic-lanceolate, slightly heteropolar, ends acute, raphe-sternum linear and straight, central area unilaterally expanded with a stigma at the opposite side, striae distintly punctuate, parallel to radiate toward the ends.

Gomphonema sp.

Figs 118-119

Valves narrowly lanceolate, heteropolar, ends acute, raphe-sternum linear and straight, central area with a stigma at the end of a stria, striae radiate, areolae inconspicuous.

Gomphonema sp. is similar to G. geisslerae Reichardt \& Lange-Bertalot in outline (lenght 18-27.5 $\mu \mathrm{m}$, width 2.6-3.8 $\mu \mathrm{m}$ ), but is wider (Reichardt 1997). 


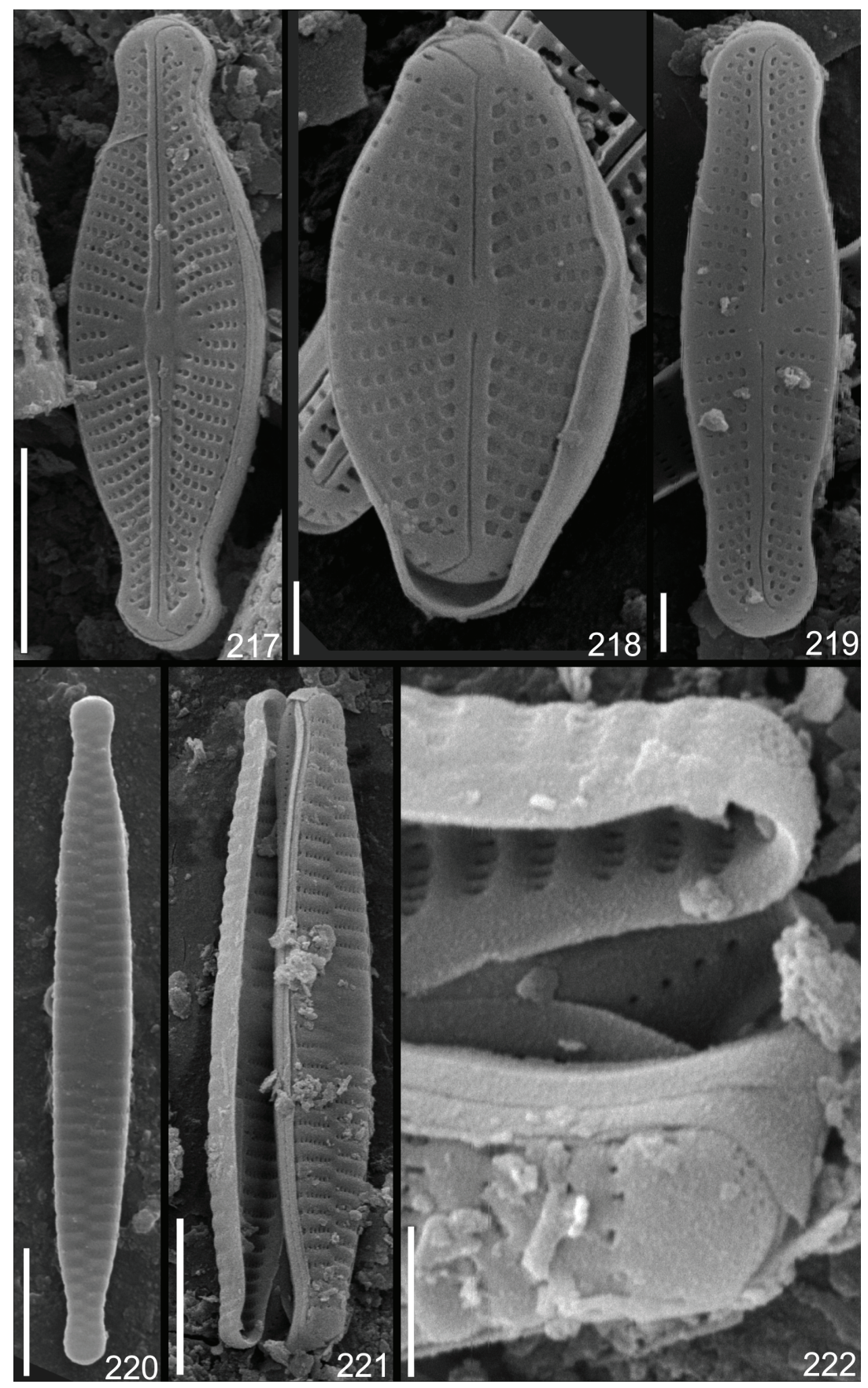

Figures 217-222. Diatoms from Piraquara II reservoir (SEM). 217. External view of Sellaphora sassiana showing the striation pattern, proximal and distal raphe ends, scale: $5 \mu \mathrm{m}$. 218. Sellaphora sardinensis in external view showing the shape of areolae, proximal and distal raphe ends, scale: $1 \mu \mathrm{m}$. 219 . Raphe valve of Achnanthidium macrocephalum in external view, scale: $1 \mu \mathrm{m}$. 220-221. Fragilaria pectinalis in external view, scales: $5 \mu \mathrm{m}$. 222. Detail of apical pore field of Fragilaria pectinalis, scale: $1 \mu \mathrm{m}$.

\section{Achnanthidiaceae}

Achnanthidium macrocephalum (Hustedt) Round \& Bukhtiyarova, Diatom Res. 11: 349, 1996.

Figs. 114-115, 219

Valves lanceolate, ends capitate, sternum narrow and linear, central area round, slightly expanded, raphe straight, striae inconspicuous.

The measurements of analysed specimens agree with the limits given by Krammer \& Lange-Bertalot (1991b) of species Achnanthes minutissima var. macrocephala Hustedt (lenght 6-14 $\mu \mathrm{m}$, width 2.5-3 $\mu \mathrm{m}$ ).
Achnanthidium reimeri (Camburn) Ponader \& Potapova present similar outline, but the valve is wider (9.4-13.5 $\mu \mathrm{m}$ long, 2.9-3.2 $\mu \mathrm{m}$ wide) and the apices are more rounded. Also, A. latecephalum Kobayasi has similar outline, but the capitate ends and parallel striae distinguish the species from A. macrocephalum (Ponader \& Potapova 2007).

\section{Amphipleuraceae}

Frustulia quadrisinuata Lange-Bertalot in Lange-Bertalot \& Metzeltin, Iconogr. Diatomol. 2: 59-60, pl. 38, figs 10-12, pl. 119, figs 1-1, 1996.

Fig. 157 
Valves lanceolate with margins slightly triondulate, ends rostrate, raphe-sternum narrow and linear, with longitudinal costa constricted at the central area, raphe straight, striae inconspicuous.

\section{Sellaphoraceae}

Sellaphora sardiniensis Lange-Bertalot, Cavacini, Tagliaventi \& Alfinito in Lange-Bertalot, Iconogr. Diatomol. 12: 122; pl. 19, fig. 1-9, pl. 20, fig. 1-5, 2003.

Figs. 149-150, 218

Valves elliptic, ends subcapitate, raphe-sternum narrow and linear, central area elliptic, limited by irregular shortening striae, raphe straight, striae radiate.

Sellaphora sardiniensis resembles S. subpupula Levkov \& Nakov, but the latter taxon have central area laterally expanded and denser striae (27-30 in $10 \mu \mathrm{m}$ ) (Lange-Bertalot et al. 2003; Levkov et al. 2007).

Sellaphora sp.

Fig. 148

Valves linear-lanceolate, ends subcapitate, raphe-sternum narrow and linear, elliptic central area limited by irregular shortening striae; raphe straight with proximal ends curved to the same side, striae radiate.

Sellaphora sp. is similar to $S$. rhombicarea Metzeltin, Lange-Bertalot \& García-Rodriguez in outline, but differs in the dimensions (lenght 24-50 $\mu \mathrm{m}$, width $9.5-11 \mu \mathrm{m}, 17-19$ striae in $10 \mu \mathrm{m}$ ) (Metzeltin et al. 2005). Sellaphora laevissima (Kützing) Mann is larger and striae less dense (lenght 6-9.3 $\mu \mathrm{m}$ and 15-19 striae in $10 \mu \mathrm{m}$ ) (Zimmerman et al. 2010).

\section{Surirellaceae}

\section{Surirella sp.}

Fig. 209

Valves isopolar, linear to lanceolate, slightly constricted in the middle, ends cuneate-rounded. Aliform chanells parallel, straight to slightly radiated near the ends, striae inconspicuous.

\section{Acknowledgments}

The authors thank CAPES (Coordenação de Aperfeiçoamento de Pessoal de Nível Superior) for the Masters and pos doctoral scholarships granted to RC Marra and PI Tremarin, respectively; to CNPq (Conselho Nacional de Desenvolvimento Científico e Tecnológico) for the research productivity scholarship awarded to TAV Ludwig. Also, we thank Dr. Maurício Bergamini Scheer for the help with fieldwork and SANEPAR (Companhia de Saneamento do Paraná) for field logistic assistance. Financial support was provided from the Araucária Foundation (5929/2011).

\section{References}

BARBER, H. \& HAWORTH, E. 1981. A guide to the morphology of the diatom frustule. Freshw. Biol. Assoc. 44: 1-112.

BERTOLLI, L., TREMARIN, P.I. \& LUDWIG, T.A.V. 2010. Diatomáceas perifíticas em Polygonum hydropiperoides Michaux, reservatório do Passaúna, Região Metropolitana de Curitiba, Paraná, Brasil. Acta Bot. Bras. 24: 1065-1081.

BITTENCOURT, S. \& GOBBI, E.F. 2006. Carga máxima de fósforo admissível ao reservatório Piraquara II, uma aplicação do processo TMDL. Rev. Bras. Ciênc. Solo 30: 595-603.

CALISTO, M., MOLOZZI, J. \& BARBOSA, J.L.E. 2014. Eutrophication of Lakes. In: Eutrophication causes, consequences and control (Ansari, A.A., Gill, S.S., eds.). Springer Netherlands. v. 2, p. 55-71.

CAMBURN, K.E. \& CHARLES, F. 2000. Diatoms of low alkalinity lakes in the Northeastern United States. J. Paleolimnol. 25: 129-130.

CATTANEO, A. \& KALFF, J. 1979. Primary production of algae growing on natural and artificial aquatic plants: a study of interactions between epiphytes and their substrate. Limnol. and Oceanogr. 24: 1031-1037.
CETTO, J.M., LEANDRINI, J.A., FELISBERTO, A.S. \& RODRIGUES, L. 2004 Comunidades de algas perifíticas no reservatório de Iraí, estado do Paraná, Brasil. Acta Sci., Biol. Sci. 26(1): 1-7.

COMPÈRE, P. 2001. Ulnaria (Kützing) Compère, a new name for Fragilaria subgen. Alterasynedra Lange-Bertalot with comments on the typification of Synedra Ehrenberg. In Lange-Bertalot Festschrift, Studies on diatoms. (R. Jahn, J.P. Kociolek, A. Witkowski, P. Compère, eds). ARG Gantner Verlag KG, Rugell, p.97-101.

COSTA, J.C.F. 1995. Diatomáceas (Bacillarophyceae) da reserva biológica de Poço das Antas, Município de Silva Jardim, Rio de Janeiro, Brasil. Iheringia, Sér. Bot. 46: 57-143.

COX, E.J. 2003. Placoneis Mereschkowsky (Bacillariophyta) revisited: resolution of several typification and nomenclatural problems, incluing the generitype. Bot. J. Linn. Soc. 141: 53-83.

FARIA, D.M. 2010. Diatomáceas perifíticas de um reservatório eutrófico do rio Itaqui: aspectos qualitativos e quantitativos. Dissertação de Mestrado, Universidade Federal do Paraná.

FARIA, D.M., TREMARIN, P.I. \& LUDWIG, T.A.V. 2010. Diatomáceas perifíticas da represa Itaqui, são José dos Pinhais, Paraná: Fragilariales, Eunotiales, Achnanthales e Gomphonema Ehrenberg. Biota Neotrop. 10(3): 415-427. http://www.biotaneotropica.org.br/v10n3/pt/fullpaper?bn04110032010+pt (retrieved on 29/07/2015)

FARIA, D.M., GUIMARÃES, A.T.B. \& LUDWIG, T.A.V. 2013. Responses of periphytic diatoms to mechanical removal of Pistia stratiotes $\mathrm{L}$. in a hypereutrophic subtropical reservoir: dynamics and tolerance. Braz. J. Biol. 73(4): 681-689.

FRENGUELLI, J. 1941. Contribuciones al conocimiento de las Diatomeas Argentinas. XVI. Diatomeas del Rio de La Plata. Rev. Mus. La Plata 3: 213-334.

HOFMANN, G., LANGE-BERTALOT, H. \& WERUM, M. 2013. Bestimmungflora Kieselalgen für die ökologische Praxis. Über 700 der häufigsten Arten und ihre Ökologie. In Diatom im Sübwasser-Benthos von Mitteleuropa (H. LangeBertalot, ed.). $908 \mathrm{p}$.

HICKEL, B. \& HÄKANSSON, H. 1991. The freshwater diatom Aulacoseira herzogii. Diatom Res. 6(2): 299-305.

HOUK, V., KLEE, R. \& TANAKA, H. 2010. Atlas of freshwater centric diatoms with a brief key and descriptions, Part III. Stephanodiscaceae A. Cyclotella, Tertiarius, Discostella. Fottea (Suppl.) 10: 1-498.

HLÚBIKOVÁ, D., ECTOR, L. \& HOFFMANN, L. 2011. Examination of the type material of some diatom species related to Achnanthidium minutissimum (Kütz.) Czarn. (Bacillariophyceae). Algol. Stud. 136/137(1): 19-43.

HUBER-PESTALOZZI, G. 1942. Das Phytoplankton des Süsswassers Systematik und Biologie (Diatomeen). In Die Binnengewässer (A. Thienemann, ed.). E SchweizerbartscheVerlagsbuchhandlung, Stuttgart, v.16, pars 2, 549 p.

HUSTEDT, F. 1961-1966. Die Kieselalgen. In Kryptogamen-Flora (L. Rabenhorst, ed.). Akademische Verlagsgesellschaft, Leipzig, v.7, pars 3, 816 p.

KOCIOLEK, J.P. 2005. Taxonomy and ecology: further considerations. Proc. Cal Acad. Sci. 56(10): 99-106.

KRAMMER, K. 1992. Pinnularia: Eine Monographie der europäischen Taxa. Biblioth. Diatomol. 26: 1-353.

KRAMMER, K. 1997a. Die cymbelloidean Diatomeen: eine Monographie der weltweit bekannten taxa. I Allgemeines und Encyonema part. Biblioth. Diatomol. 36: 1-382.

KRAMMER, K. 1997b. Die cymbelloidean Diatomeen: eine Monographie der weltweit bekannten taxa. II Encyonema part., Encyonopsis and Cymbellopsis. Biblioth. Diatomol. 37: 1-469.

KRAMMER, K. 2000. The genus Pinnularia. In Diatoms of Europe, Diatoms of the European Inland waters and comparable habitats (H. Lange-Bertalot, ed.). A.R.G. Gantner Verlag KG, Ruggell, v.1, 703 p.

KRAMMER, K. 2002. Cymbella. In Diatoms of Europe, Diatoms of the European Inland waters and comparable habitats (H. Lange-Bertalot, ed.). A.R.G. Gantner Verlag KG, Ruggell, v. 3, 584 p.

KRAMMER, K. 2003. Cymbopleura, Delicata, Navicymbula, Gomphocymbellopsis, Afrocymbella. In Diatoms of Europe, Diatoms of the European Inland waters and comparable habitats (H. Lange-Bertalot, ed.). A.R.G. Gantner Verlag KG, Ruggell, v. 4, 584 p. 
KRAMMER, K. \& LANGE-BERTALOT, H. 1986. Bacillariophyceae: Naviculaceae. In Süsswasserflora von Mitteleuropa (H. Ettl, J. Gerloff, H. Heynig, D. Mollenhauer, eds). Gustav Fischer, Jena. v. 2, pars 1, 876 p.

KRAMMER, K. \& LANGE-BERTALOT, H. 1988. Bacillariophyceae: Bacillariaceae, Epithemiaceae, Surirellaceae. In Süsswasserflora von Mitteleuropa (H. Ettl, J. Gerloff, H. Heynig, D. Mollenhauer, eds). Gustav Fischer, Stuttgart. v. 2,pars 2, $596 \mathrm{p}$.

KRAMMER, K. \& LANGE-BERTALOT, H. 1991a. Bacillariophyceae: Centrales, Fragilariaceae, Eunotiaceae. In Süsswasserflora von Mitteleuropa (H. Ettl, J. Gerloff, H. Heynig, D. Mollenhauer, eds). Gustav Fischer, Stuttgart. v. 2, pars 3, $576 \mathrm{p}$.

KRAMMER, K. \& LANGE-BERTALOT, H. 1991b. Bacillariophyceae: Achnanthaceae. Kritische Ergänzungen zu Navicula (Lineolatae) und Gomphonema. In Süsswasserflora von Mitteleuropa (H. Ettl, J. Gerloff, H. Heynig, D. Mollenhauer, eds). Gustav Fischer, Stuttgart. v. 2, pars 4, 437 p.

KULIKOVSKIY, M.S., LANGE-BERTALOT, H., WITKOWSKI, A., DOROFEYUK, N.I. \& GENKAL, S.I. 2010. Diatom assemblages from Sphagnum bogs of the world. Biblioth. Diatomol 55:1-326.

KULIKOVSKIY, M., GUSEV, E., ANDREEVA, S. \& ANNENKOVA, N. 2014. Phylogenetic position of the diatom genus Geissleria Lange-Bertalot \&Metzeltin and description of two new species from Siberian mountain lakes. Phytotaxa 177: $249-260$

LANGE-BERTALOT, H. \& METZELTIN, D. 1996. Oligotrophie indikatoren: 800 Taxa repräsentativ für drei diverse see-typen, kalkreich-oligodystroph-schwach gepuffertes weichwasser. In Iconogr. Diatomol., Annotated Diatom Monographs (H. Lange-Bertalot, ed.). Gantner Verlag KG, Ruggell, v.2, 390 p.

LANGE-BERTALOT, H. \& MOSER, G. 1994. Brachysira, Monographie der Gattung. Biblioth. Diatomol. 29: 1-212.

LANGE-BERTALOT, H., BAK, M., WITKOWSKI, A. \& TAGLIAVENTI, N. 2011. Eunotia and some related genera. In Diatoms of Europe, Diatoms of the European Inland waters and comparable habitats (H. Lange-Bertalot, ed.). Gantner Verlag KG, Ruggell, v. 6, 747 p.

LANGE-BERTALOT, H., CAVACINI, P., TAGLIAVENTI, N. \& ALFINITO, S. 2003. Diatoms of Sardinia. In Iconogr. Diatomol., Annotated Diatom Monographs (H. Lange-Bertalot ed.). Gantner Verlag KG, Ruggell, v.12, 438 p.

LEVKOV, Z., KRSTIC, S., METZELTIN, D. \& NAKOV, T. 2007. Diatoms of Lakes Prespa and Ohrid: about 500 taxa from ancient lake system. In Iconogr. Diatomol., Annotated Diatom Monographs (H. Lange-Bertalot, ed.). Gantner Verlag KG, Ruggell, v.16, 613 p.

LOWE, R.L., PATRICK, K., JOHANSEN, J.R., VAN DE VIJVER, B., LANGEBERTALOT, H. \& KOPALOVÁ, K. 2014. Humidophila gen. nov., a new genus for a group of diatoms (Bacillariophyta) formely within the genus Diadesmis: species from Hawaii, incluing one new species. Diatom Res. 29(4): 351-360.

LUDWIG, T.A.V. \& VALENTE-MOREIRA, I.M. 1990. Contribuição ao conhecimento da diatomoflórula do parque regional do Iguaçu, Curitiba, Paraná, Brasil: II. Cêntricas (Bacillariophyceae). Braz. Arch. Biol. Tecnol. 33(4): 843-852.

MANN, D.G., MACDONALD, S.M., BAYER, M.M., DROOP, S.J.M., CHEPURNOV, V.A., LOKE, R.E., CIOBANU, A. \& DU BUF, J.M.H. 2004. The Sellaphora pupula species complex (Bacillariophyceae): morphometric analysis, ultrastructure and mating data provide evidence for five new species. Phycol. 43(4): 459-482.

MATEUCCI, S.D. \& COLMA, A. 1982. La metodología para estudo de la vegetación. Collecion de Monografías Científicas, n. 22, p.168.

MELTZELTIN, D. \& LANGE-BERTALOT, H. 1998. Tropical Diatoms of South America I. About 700 predominantly rarely known or new taxa representative of the neotropical flora. In Iconogr. Diatomol., Annotated Diatom Monographs (H. Lange-Bertalot ed.). Gantner Verlag KG, Ruggell, v. 5, 220 p.

MELTZELTIN D. \& LANGE-BERTALOT, H. 2007. Tropical Diatoms of south America II. Special remarks on biogeographic disjunction. In Iconogr. Diatomol., Annotated Diatom Monographs (H. Lange-Bertalot ed.). Gantner Verlag KG, Ruggell, v. 18, 1876 p.

MELTZELTIN D. \& LANGE-BERTALOT, H. 2002. Diatoms from the Island Continent Madagascar. In Iconogr. Diatomol., Annotated Diatom Monographs (H. Lange-Bertalot ed.). Gantner Verlag KG, Ruggell, v. 11, 286 p.

MELTZELTIN, D., LANGE-BERTALOT, H. \& GARCÍA-RODRÍGUEZ, F. 2005. Diatoms of Uruguay compared with other taxa from South America and elsewhere. In Iconogr. Diatomol., Annotated Diatom Monographs (H. LangeBertalot ed.). Gantner Verlag KG, Ruggell, v.5, 736 p.

MOREIRA-FILHO, H. \& VALENTE-MOREIRA, I.M. 1981. Avaliação taxonômica e ecológica das diatomáceas (Bacillariophyceae) epífitas em algas pluricelulares obtidas nos litorais dos estados do Paraná, Santa Catarina e São Paulo. Bol. Mus. Bot. Mun., Curitiba 47: 1-17.

PATRICK, R. \& REIMER, C.W. 1966. The diatoms of United States: exclusive of Alaska and Hawaii. Acad. Nat. Sci. Philadelphia, Monographs, n.13. v. 1, 688 p.

PATRICK, R. \& REIMER, C.W.1975. The diatoms of the United States: exclusive of Alaska and Hawaii. Acad. Nat. Sci. Philadelphia, Monographs, n.13. v. 2, 213 p.

PONADER, K.C. \& POTAPOVA, M.G. 2007. Diatoms from the genus Achnanthidium in flowing waters of the Appalachian Mountains (North America): ecology, distribution and taxonomic notes. Limnologica 37: 227-241.

POTAPOVA, M. 2009. Achnanthidium minutissimum. In Diatoms of the United States. http://westerndiatoms.colorado.edu/taxa/species/Achnanthidium_minutissimum (retrieved on 01/06/2015)

REICHARDT, E. 1995. Die Diatomeen (Bacillariophyceae) in Ehrenbergs Material von Cayenne, Guayana Gallica (1843). In Iconogr. Diatomol., Annotated Diatom Monographs (H. Lange-Bertalot, ed.). Iconogr. Diatomologica. Gantner Verlag KG, Ruggell, v. 1, 49 p.

REICHARDT, E. 1997. Taxonomische Revision dês Artenkomplexes um Gomphonema pumilum (Bacillariophyceae). Nova Hewigia 65: 114-115.

REICHARDT, E. 2015. Gomphonema gracile Ehrenberg sensu stricto et sensu auct. (Bacillariophyceae): a taxonomic revision. Nova Hedwigia 101: 367-393.

REICHARDT, E. 2005. Gomphonema dubravicense Pantocsek eine taxonomische Revision. Diatom Res. 20(2): 335-349.

ROUND, F.E. \& BASSON, P.W. 1997. A new monoraphid diatom genus (Pogoneis) from Bahrain and the transfer of previously described species A. hungarica and A. taeniata to new genera. Diatom Res. 12(1): 71-81.

ROUND, F.E., CRAWFORD, R.M. \& MANN, D.G. 1990. The diatoms: biology e morphology of the genera. Cambridge, University Press. 747 p.

RUMRICH, U., LANGE-BERTALOT, H. \& RUMRICH, M. 2000. Diatoms of the Andes. From Venezuela to Patagonia/Tierra del Fuego and two additional contributions. In Iconogr. Diatomol. Annotated Diatom Monographs (H. LangeBertalot, ed.). ARG Gantner Verlag KG, Rugell, v. 9, 673 p.

SILVA, A.M., LUDWIG, T.A.V., TREMARIN, P.I. \& VERCELLINO, I.S. 2010. Diatomáceas perifíticas em um sistema eutrófico brasileiro (Reservatório do Iraí, estado do Paraná). Acta Bot. Bras. 24(4): 997-1016.

SIMONSEN, R. 1974. The diatom plankton of Indian Ocean Expedition of R/V "Meteor", 1964-65 Meteror Forschungsergebnisse. Reihe D-Biologie 19: 1-66.

SIMONSEN, R. 1987. Atlas and catalogue of the diatom types of Friedrich Hustedt. Gebrüder Nortraeger Versbuchanandlung. J. Cramer, Stuttgart. 3 v.

SIVER, P.A. \& HAMILTON, P.B. 2011. The freshwater flora of waterbodies on the Atlantic Coastal Plain. In Iconogr. Diatomol., Annotated Diatom Monographs (H. Lange-Bertalot, ed.). ARG Gantner Verlag KG, Rugell, v. 22: 1-916.

SIVER, P.A. \& KLING, H. 1997. Morphological observations of Aulacoseira using scanning electron microscopy. Can. J. Bot. 75: 1807-1835.

STOERMER, E.F. \& SMOL, J.P. 2004. The Diatoms: application for the environmental and earth sciences. Cambridge University Press (reimpr.). 469 p.

TAYLOR, J.C., HARDING, W.R. \& ARCHIBALD, G.M. 2007. An illustrated guide to some common diatom species from South Africa. WRC Report TT 282/07. Pretoria. Water Research Commission. 282 p.

TORGAN, L.C. \& OLIVEIRA, M.A. 2001. Geissleria aikenensis (Patrick) Torgan et Oliveira comb. nov.: morphological and ecological characteristics. In Proceeding of 16th International Diatom Symposium (A. Economou-Amilli, ed.). Amvrosiou Press, Athens, p. 115-125.

TREMARIN, P.I., LUDWIG, T.A.V. \& TORGAN, L.C. 2012. Ultrastructure of Aulacoseira brasiliensis sp. nov. (Coscinodiscophyceae) and comparison with related species. Fottea 12(2): 171-188.

TREMARIN, P.I., LUDWIG, T.A.V., TORGAN, L.C. 2014. Four new Aulacoseira species (Coscinodiscophyceae) from Matogrossense Pantanal, Brazil. Diatom Res. 29(2): 183-199. 
TREMARIN, P.I., STRAUBE, A. \& LUDWIG, T.A.V. 2015. Nupela (Bacillariophyceae) in littoral rivers from south Brazil, and description of six species of the genus. Fottea 15(1): 77-93.

TREMARIN, P.I., KIM, K.M.I., MARRA, R.C. \& LUDWIG, T.A.V. 2016. Additional data on morphology of Actinella leontopithecus-rosalia Costa (Bacillariophyta, Eunotiaceae). Phytotaxa 247(4): 259-266.

TUJI, A. \& WILLIAMS, D.M. 2006. Examination of the type Synedra rumpens $=$ Fragilaria rumpens, Bacillariophyceae. Phycol. Res. 54: 99-103.

TUJI, A. \& WILLIAMS, D.M. 2008a. Examination of types in the Fragilaria pectinalis- capitellata species complex. In Proceedings of the 19th International Diatom Symposium (Y. Likhoshway, ed.). Biopress Limited, Bristol, p. 125-139.

TUJI, A., WILLIAMS, D.M. 2008b. Examination of types material in the Fragilaria mesolepta Rabenhorst and two similar, but distinct, taxa. Diatom Res. 23(2): 503-510.

TUJI, A., WILLIAMS, D.M. 2008c. Typification abd type examination of Synedra familiaris Kütz. and related taxa. Diatom 24: 25-29.

VAN HEURCK, H. 1880. Synopsis des Diatomées de Belgique. Anvers.

WETZEL, C.E. \& ECTOR, L. 2015. Taxonomy and ecology of Fragilaria microvaucheriae sp. nov. and comparison with the type materials of $F$. uliginosa and F. vaucheriae. Cryptogamie, Algol. 36(3): 271-289.

WETZEL, C.E., ECTOR, L., VAN DE VIJVER, B., COMPÈRE, P. \& MANN, D.G. 2015. Morphology, typification and critical analysis of some ecologically important small naviculoid species (Bacillariophyta). Fottea 15(2): 203-234, 396 figs, 1 table.
WETZEL, C.E., MORALES, E.A., HINZ, F., BICUDO, D.C. \& ECTOR, L. 2013 a Fragilariforma javanica comb. nov.: analysis of type material of a widely reported species with a tropical distribution. Diatom Res. 28(4): 373-379.

WETZEL, C.E., VAN DE VIJVER, B., HOFFMAN, L. \& ECTOR, L. 2013b. Planothidium incuriatum sp.nov. a widely distributed diatom species (Bacillariophyta) and type analysis of Planothidium biporomum. Phytotaxa. 138(1): 43-57.

WOJTAL, A., ECTOR L., VAN DE VIJVER, B., MORALES, E.A., BLANCO, S., PIATEK, J. \& SMIEJA, A. 2011. The Achnanthidium minutissimum complex (Bacillariophyceae) in southern Poland. Algol. Stud. 136/137:211-238.

ZELAZNA-WIECZOREK, J. 2011. Diatom flora in springs of Lódz Hills (Central Poland): biodiversity, taxonomy, and temporal changes of epipsammic diatom assemblages in springs affected by human impact. Diatom Monographs, v.13, p.1-419.

ZIMMERMANN, C., POULIN, M. \& PIENITZ, R. 2010. Diatoms of North American: The Pliocene-Pleistocene freshwater flora of Bylot Island, Nunavut, Canadian High Arctic. In Iconogr. Diatomol., Annotated Diatom Monographs (H. Lange-Bertalot ed.). Gantner Verlag KG, Ruggell, v. 21, p.1-405.

Received: 26/04/2016

Revised: 23/09/2016

Accepted: 07/10/2016 